\title{
Localized deformation induced by heterogeneities in porous carbonate analysed by multi-scale digital image correlation
}

\author{
Jérémie Dautriat ${ }^{\mathrm{a}, *}$, Michel Bornert ${ }^{\mathrm{b}}$, Nicolas Gland ${ }^{\mathrm{a}}$, Alexandre Dimanov ${ }^{\mathrm{c}}$, Jean Raphanel ${ }^{\mathrm{c}}$ \\ a IFP Energies nouvelles, Ingénierie de Réservoir, $1 \mathcal{E} 4$ av. de Bois-Préau, 92852 Rueil-Malmaison Cedex, France \\ b Laboratoire Navier, Ecole des Ponts-ParisTech, Université Paris Est, 6-8 av. Blaise Pascal, 77455 Marne-la-Vallée Cedex 2, France \\ ' Laboratoire de Mécanique des Solides, Ecole Polytechnique ParisTech, 91128 Palaiseau, France
}

\begin{abstract}
The understanding and the prevention of damage mechanisms, which have an impact on the hydrocarbon production and recovery rates, are of paramount interest for reservoir engineers. The modelling of such coupled processes relies essentially on experimentally obtained data, which characterize the macroscopic mechanical and transport properties. This approach however cannot account for the multi-scale structural heterogeneities of the considered rocks, in spite of their fundamental importance. The microstructural characterization of damage is usually based on 'post-mortem' observations of the samples, which provide both qualitative and quantitative information about the effects of the mechanisms activated at the grain scale and at intermediate scales, at a pervasive stage of damage after sample unloading. New techniques provide more quantitative and direct methods to follow the deformation history and the eventual development of localization and damage. In this study, the 2D Digital Image Correlation (DIC) technique has been applied to sequences of images taken from carbonate samples during uniaxial compression tests. Several scales have been considered, ranging from the centimetric scale of the samples to the local scale of their microstructure. For this purpose both optical observations and Scanning Electron Microscopy (SEM) were used. Although the macroscopic strain at failure was very small $(<0.2 \%)$, the DIC technique has proven to be reliable, provided one selects carefully image acquisition conditions and DIC parameters, as highlighted in our discussion on the uncertainties and the evaluation of errors. This technique has allowed us to quantify both the global and local strain fields during the deformation process. We have thus been able to precisely identify the localizations of damage and the local compaction mechanisms, and to relate them to the characteristic structural heterogeneities of the tested carbonate.
\end{abstract}

\section{Introduction}

Experimental investigations of petrophysical and mechanical properties of rocks are commonly performed at a macroscopic scale. In the laboratory, the behavior of a specimen is first globally characterized by means of macroscopic measurements of physical properties (conductivity, permeability, porosity...) and mechanical constants (compressibility, elastic moduli, yield strength...). "Postmortem" microstructural investigations may be performed in order to identify the micro-physical mechanisms responsible for the macroscopical sample response. Still, the macroscopic measurements are essentially averages, and hence the corresponding constitutive relations rely implicitly on the assumption of the homogeneity of microstructures, properties and mechanical responses. However, geomaterials present naturally very important and multi-scale structural heterogeneities, which may strongly influence the local

\footnotetext{
* Corresponding author. Tel.: +33147526758.

E-mail address: jeremie.dautriat@ifpenergiesnouvelles.fr (J. Dautriat).
}

responses to externally applied stresses, and hence induce important variability of sample-to-sample macroscopic behavior. In contrast with global and averaged measurements, specific techniques referred as full-field measurements have been developed. They provide local quantitative information on a given property, which can be correlated to a pertinent scale of heterogeneity.

One of the most widespread full-field measurement techniques is the method of displacement field measurement using Digital Image Correlation (DIC). This procedure is based on the comparison of images acquired at different stages of a mechanical test and provides qualitative and quantitative descriptions of local responses, which allow the evaluation of the impact of material heterogeneity on the strain distribution and localization (Doumalin et al., 2003; Elbachiri et al., 2008). Since the pioneer works of Peters and Ranson (1982), Sutton et al. (1983) and Chu et al. (1985), the powerful technique of 2D-DIC has been extensively applied in the research field of mechanics of solids (Sutton et al., 2009), and to various classes of materials such as metals and alloys (Vacher et al., 1999; Wattrisse et al., 2001; Tatschl and Kolednik, 2003; Pires et al., 2004; Marya et al., 2006; Héripré et al., 2007), ceramics (Huon et al., 2007), composites 
(Grédiac, 2004; Rae et al., 2004) subjected to various mechanical loadings, or physico-chemical reactions. In addition to 2D-DIC, macroscopic stereo imaging techniques give access to out-of-plane components of displacement (Sutton et al., 2008). More recently, with the fast development of 3D X-Ray (XR) tomography imaging techniques, DIC has also been extended to 3D volumetric image correlation (Bay et al., 1999; Bornert et al., 2004; Lenoir et al., 2007). Volumetric DIC is a natural extension of 2D-DIC to 3D images, obtained for instance from optical serial sectioning (Germaneau et al., 2007), by X-Ray Computed Tomography (CT) or X-Ray MicroComputed Tomography (MCT) (Hild et al., 2009) or by any alternative 3D imaging techniques. Sutton et al. (2009) report a wide review of Image Correlation techniques and applications.

In the present work, 2D-DIC is applied at different scales of observation of the heterogeneities of a natural carbonate. The acquisition of images from a sample surface has been performed by a macroscopic optical camera (Sutton et al., 1983) and optical microscopy (Schreier et al., 2004), simultaneously on a same sample (Bornert et al., 2010) or by Scanning Electron Microscopy (SEM) (Doumalin and Bornert, 2000; Doumalin et al., 2003; Héripré et al., 2007).

In the field of experimental rock mechanics, 2D-DIC measurements have recently become widespread and have been successfully applied to unconsolidated sands/soils (Gudehus and Nübel, 2004; Rechenmacher and Finno, 2004; Adam et al., 2005; Rechenmacher, 2006; Hall et al., 2009), soft rocks (Bandhari and Indue, 2005), clayrocks (Bornert et al., 2010) and to the study of crack propagation in pre-damaged volcanic tuff samples (NGuyen et al., 2011). Louis et al. (2007) have characterized the average strain and the localized compaction bands in sandstone samples by the comparison of radiographs of the specimen before and after triaxial compression. Lenoir et al. (2007) studied the initiation and the propagation of shear bands on clay-rock samples subjected to triaxial stress by applying Volumetric DIC to reconstructed volumes obtained from Synchrotron MCT.

In a previous study (Dautriat et al., submitted for publication), hydromechanical measurements under deviatoric stress conditions have been performed and post-mortem observations (SEM, XR-MCT) and characterizations (Mercury Porosimetry, NMR) have shown that the microstructural heterogeneities of the Estaillades limestone are related to the activation of local damage mechanisms affecting the evolutions of permeability and compressibility. The pertinent results in terms of loading conditions will be briefly recalled to highlight the impact of the structural heterogeneities on the mechanical behavior of this carbonate. In the present study, we have applied 2D-DIC on Estaillades limestone specimens deformed solely by uniaxial compression tests. Since this limestone exhibits several scales of heterogeneity, multi-scale observations are required. On the one hand, a set-up made of two optical cameras equipped with suitable macroscopic or microscopic optics has been used to measure the evolution of the 2D strain field at the macroscopic and mesoscopic scales on conventional macroscopic samples. This set-up is derived from the one developed by Bornert et al. (2010).

On the other hand, compression tests were realized inside the chamber of an environmental SEM, in order to measure the 2D strain field at the microscopic scales on the surface of small parallelepipedic samples (Doumalin et al., 1999).

Up to recently in rock mechanics, because of its limitations in terms of resolution, the DIC technique has rather been used for geomaterials prone to moderate to large deformation (i.e., about $1 \%$ to almost $10 \%$ of global strain) to characterize the macroscopic mechanical responses of samples (Viggiani and Hall, 2008). As reviewed by Viggiani and Hall (2008), DIC has also been used to study the propagation of fractures in geomaterials sustaining relatively small strains before failure, but generally pre-damaged with artificially induced localization. In the present case, DIC technique is used on a completely intact reservoir rock, which sustains very low strains before failure $(\varepsilon \mathrm{a}<0.2 \%)$, in order to detect localized deformation mechanisms at the local scale, taking place all along the loading and not strictly at a pervasive stage of the damage. Since error assessment is crucial to correctly quantify the very small strains, an important part of this paper is dedicated to the estimation of uncertainties with regard to the image acquisition and processing technique.

\section{Material characterization and scales of heterogeneity}

The Estaillades Limestone is a moderately porous and permeable carbonate of granular microstructure, composed almost exclusively of calcite (99\%) micrograins. These micrograins with sizes from 1 to $10 \mu \mathrm{m}$ form two kinds of aggregates, either highly dense or microporous, their sizes ranging from $100 \mu \mathrm{m}$ to $500 \mu \mathrm{m}$, as shown on Fig. 1a and b. Consequently, the porosity is bimodal, with larger porosity located between the aggregates and smaller intra-aggregate microporosity. As revealed by Mercury Porosimetry (Fig. 1c), the two peaks of the capillary pore throats' radii distribution are at $10 \mu \mathrm{m}$ and $0.2 \mu \mathrm{m}$ respectively. Hereafter, the previously described heterogeneity, observed at this microstructure scale, will be referred to as the "microheterogeneity".

At the macroscopic scale of a plug, this carbonate presents also some degree of heterogeneity, inferred from the porosity data. Indeed, the average porosity measured on 29 samples and determined by dry and saturated weights, is $28 \%$, but it varies from one plug to another from $24 \%$ up to $31 \%$ (Dautriat et al., submitted for publication). The fluctuation of the porosity between the different samples will be referred to the "macroheterogeneity". Using X-Ray tomodensitometry images inferred from medical scanner imaging and calibration curves we calculate porosity profiles along the samples and relate them to the heterogeneous distribution of both kinds of aggregates. Clusters of centimetric size could induce strong fluctuations of the porosity along the sample length, and define in the present study the "mesoheterogeneity".

As a consequence of meso and macro heterogeneities, the permeability evaluated at the scale of the samples varies as well from 50 to $150 \mathrm{mD}$, with an average value of $125 \mathrm{mD}$ (Dautriat et al., submitted for publication). The mesoheterogeneity precludes one to relate global porosity to permeability evaluated at this centimetric scale.

\section{DIC applied to the determination of deformation fields}

In this study, 2D-DIC method has been used to determine the displacement and strain fields at different scales, without contact or modification of the observed surface. This method is based on the comparison of a reference image with a current image at a deformed stage. The DIC processing follows two stages for each selected deformation level. The first stage of this comparison provides the displacement of selected positions in the reference image, regularly spaced on a grid. The second stage is the computation of the corresponding strain field relative to a chosen local gage length and/ or the computation of average strains over selected areas, including the whole sample when overall macroscopic strains are to be evaluated.

\subsection{Displacement field measurements}

The reference image is first discretized into a grid of points. To each point of index $i$ is associated a correlation window $D$. The size of the windows is kept constant for all points and for each sequence of images, and chosen according to the available local contrast and spatial resolution of the used imaging technique. The DIC procedure consists in finding the most similar domain in the 'deformed' image, by optimizing a correlation coefficient (Chu et al., 1985). For one given 

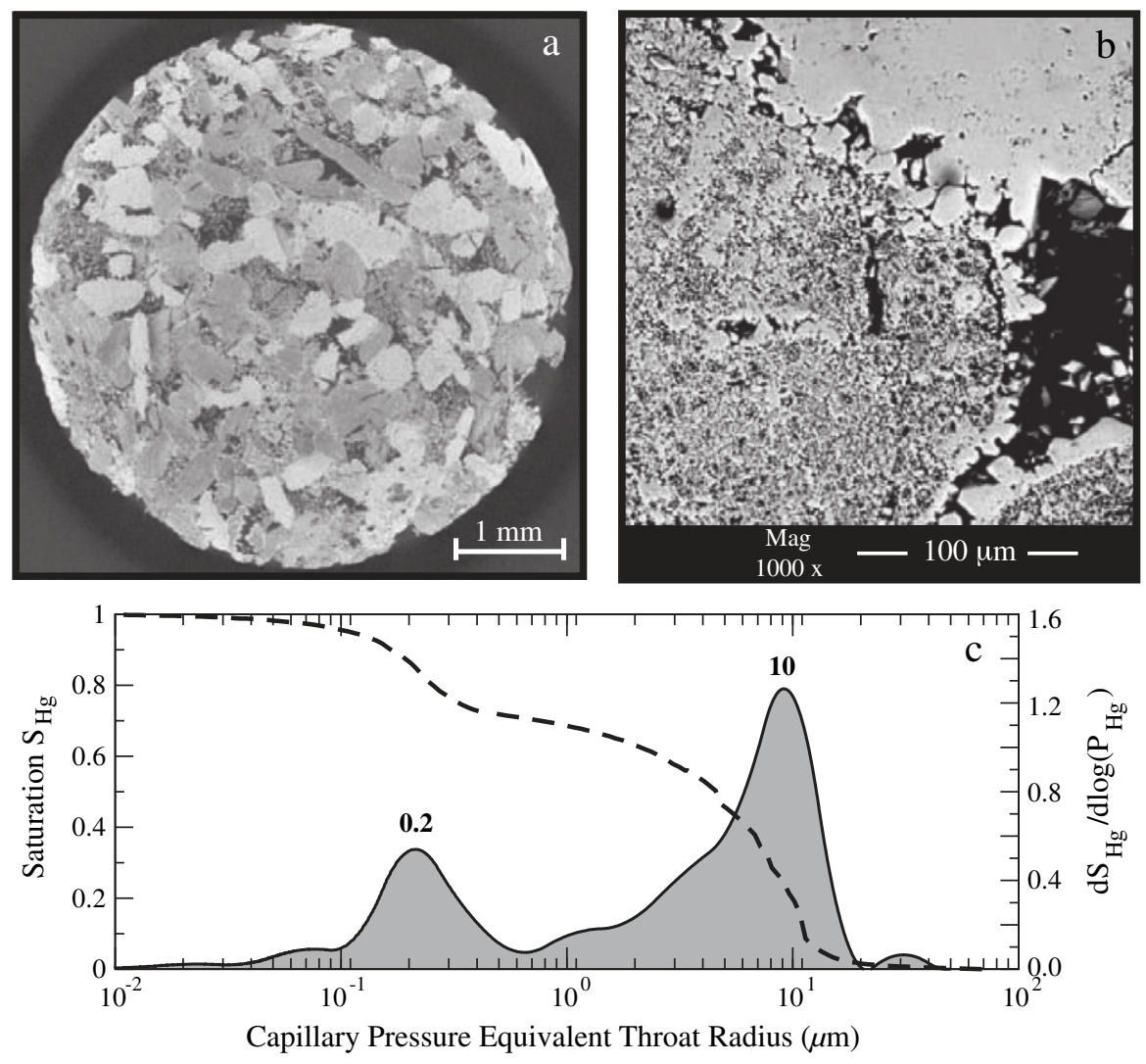

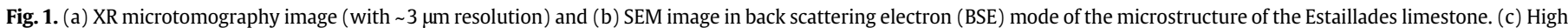
pressure mercury injection (from $0.01 \mathrm{MPa}$ to $400 \mathrm{Mpa}$ ) and porosimetry on Estaillades limestone.

domain $D$, this coefficient $C$ measures the similarity of the grey level distribution in the reference image and in the deformed image, back convected by the transformation $\phi_{0}$. The optimal transformation $\phi_{o p t}$ is used as an evaluation of the actual unknown mechanical transformation $\phi$. The method relies essentially on the assumption that the local grey level distribution is convected by the transformation $\phi$. Various choices for $C$ and $\phi_{0}$ are possible. In the present study the same definition as in Doumalin et al. (2003) has been used for the correlation coefficient $C$, expressed as:

$C\left(\phi_{0}\right)=1-\frac{\sum_{i \in D}\left(f\left(X_{i}\right)-\overline{f_{D}}\right) \cdot\left(g\left(x_{i}\right)-\overline{g_{D}}\right)}{\sqrt{\sum_{i \in D}\left(f\left(X_{i}\right)-\overline{f_{D}}\right)^{2}} \cdot \sqrt{\sum_{i \in D}\left(g\left(x_{i}\right)-\overline{g_{D}}\right)^{2}}}$,

where $X_{i}$ and $x_{i}=\phi_{0}\left(X_{i}\right)$ are respectively the coordinates (in pixels) of homologous points in the reference image and in the deformed image relative to the transformation $\phi_{0} . f(X i)$ and $g(x i)$ are respectively the grey levels of point $i$ in the reference image and in the deformed image. Positions in the reference image coincide with pixel centers, while in the deformed image their coordinates might not be integers. Finally, $\overline{f_{d}}$ and $\overline{g_{d}}$ are respectively the averages of the grey levels on the domain $D$ and on the homologous domain transformed by $\phi_{0}$. $C$ varies from 0 for perfect match, to a maximum of 2 when $f$ and $g$ present opposite contrast, the value 1 corresponding to no match at all. This measure of similarity is insensitive to global contrast or brightness variations inside the domain before and after transformation, which are often observed, especially for SEM imaging, and is therefore more robust than other correlation coefficients. The restrictive condition for the efficiency of DIC is that images must present enough contrast to generate sufficient grey level fluctuations inside each correlation window $D$.
In the simplest formulation, the approximated local transformation $\phi_{0}$ is taken as a rigid translation, which requires the evaluation of two components. It might be improved by considering as well a rotation and a homogeneous deformation, or even higher order transformations, at the cost of having additional parameters to optimize. In the present implementation, a mixed iterative formulation is used, in which $C$ is optimized only with respect to the translation components while the deformation gradient components are held fixed, but might be non null. They are set to be equal to either the overall deformation gradient or a local gradient, computed, at iteration $p$, from the displacement of neighboring measurement positions evaluated after iteration $p-1$. Such a procedure turns out to be more stable than a direct optimization of all parameters and is sufficient, especially in the present case where strains are very small so that higher order terms are less critical. We refer to Bornert et al. (2009) for a more detailed discussion on the relative importance of the form of the shape function $\phi_{0}$ with respect to accuracy on displacement components. The optimization of the correlation coefficient is performed in an automated way by the in-house software "CMV" (CorrelManuV., 2005). Furthermore, local in-plane displacements are evaluated with a sub-pixel resolution by means of bi-linear or bi-cubic interpolations of the grey level $g\left(x_{i}\right)$ in the deformed image. The choice of one or the other scheme is made in order to reduce random and systematic errors as described in Section 5.

\subsection{Strain field computation}

Once the displacement of each grid point is known, the local strain field and overall strains can be computed. Because of the multi-scale nature of the mechanical transformation and its possible discontinuities, the standard mathematical definition of deformation gradient, as the derivative of the displacement cannot be applied in a straightforward way to the actual material. It has to be replaced by 
some finite difference relative to a chosen gage length. Among various possible definitions, we retain the following one; at a given point, the strain is defined as the average of the transformation gradient on a domain $\Omega$ surrounding the considered point (Allais et al., 1994), the size of which determines the local gage length and can be freely selected:

$$
\underline{\underline{F}}^{\Omega}\left(\underline{X}_{i}\right)=\left|\frac{\partial \underline{\phi o p t}}{\partial \underline{X}}\right|_{\Omega\left(X_{i}\right)}
$$

In practice and for $2 \mathrm{D}-\mathrm{DIC}$, the domain $\Omega$ is delimited by neighboring measurement points, where the transformation $\phi_{\text {opt }}$ has been evaluated by the DIC technique, as previously described. A simple Green's formula allows one to compute this average from the displacement of the surrounding points only, whatever the displacement inside $\Omega$, by means of a contour integration. A linear interpolation of the displacement field between neighboring positions is used for simplicity to perform this calculation.

In-plane displacement fields provided by 2D-DIC are not the most pertinent quantity to detect local deformation mechanisms. Local strains are actually a much better signature of the ongoing mechanisms. In-plane components of the strain tensor can be obtained from the in-plane components of displacements, as long as the strains remain small, and out-of-plane rigid body motions of the sample, with respect to fixed reference frame of the camera, can be neglected.

As only very small strains are considered in this study, this condition is satisfied and sufficient to evaluate the in-plane components of the strain tensor. This has been verified in our experiments except for some tests during which overall deformation gradients due to an overall tilt of the sample (probably caused by non perfectly parallel faces) has been observed. But such situations can be detected from the analysis of the overall shape of the strain field. A general procedure to deal with out-of-plane motions would require the use of stereo-correlation techniques. These are however hard to apply in our situation because of the limited depth of field of our optical set-ups. The quantification of the errors due to an overall uniform out-of-plane motion is further discussed in Section 5. Additional general discussions on these aspects can be found in Sutton et al. (2008) and an ad-hoc improvement of the present set-up is described by Yang et al. (2010).

In this study, the local strain is obtained by the integration on a square perimeter defined by the eighth closest neighboring points; this configuration provides a suitable compromise between accuracy and spatial resolution, in combination with the choice of the grid step.

Special emphasis will be given to the equivalent von Mises strain, defined here as $\varepsilon_{\mathrm{eq}}=2 / 3\left|\varepsilon_{1}-\varepsilon_{2}\right|$, where $\varepsilon_{1}$ and $\varepsilon_{2}$ are the principal inplane strains. Note that this definition coincides with the 3D-von Mises equivalent strain when the transformation has axial symmetry with respect to the first principal axis; it is thus the signature of the existence of local shear deformation.

In addition to local strains, one can also compute average strains over any domain $\Omega$, by means of Eq. (2) and the same procedure. In particular, when the domain $\Omega$ extends over the whole region of interest, overall average strains are obtained from a contour integration around the whole grid. When the region of interest encompasses the whole specimen, the results are the in-plane components of the macroscopic strain tensor, which can be compared to standard macroscopic measurements.

\section{Experimental set-ups and procedures}

Three main set-ups and procedures for mechanical testing have been used on samples of Estaillades carbonate. The first set of mechanical tests has been performed with a triaxial cell described by Dautriat et al. (2009), which allows permeability measurements in three orthogonal directions. Pertinent results will be presented in this section, in order to highlight the impact of the multi-scale structural heterogeneities of this carbonate on its hydromechanical behavior. The two other sets of mechanical tests are unconfined compression tests allowing direct observations of the sample surfaces during loading. On the one hand, cylindrical samples of about $100 \mathrm{~mm}$ in height and $50 \mathrm{~mm}$ in diameter are deformed in a compression loading device, equipped with cameras and optical devices positioned on both sides of the sample allowing one to record images at both global and local scales simultaneously. On the other hand, smaller parallelipedic samples of about $10 \mathrm{~mm}$ in height and $5 \mathrm{~mm}$ in width are deformed in the chamber of an SEM and secondary electrons (SE) images are recorded with different magnifications and from different areas.

\subsection{Results of previous compression experiments using a triaxial cell}

In Fig. 2, we present a hydromechanical characterization of brine saturated Estaillades samples under triaxial loading with constant confining pressure of $3 \mathrm{MPa}$ (the minimum required to ensure core sleeve sealing for the purpose of permeability measurements). We have deformed three Estaillades samples of porosity ranging from $26.3 \%$ to $28.6 \%$, at low axial loading rate $(0.1 \mathrm{MPa} / \mathrm{min}$, corresponding to an axial strain rate of $\sim 5.10-7 s-1$ ), in drained conditions and at constant pore fluid pressure of $1 \mathrm{MPa}$. Two external LVDT sensors and a double cantilever beam extensometer record axial and radial displacements respectively. The measured Young's moduli range from $5 \mathrm{GPa}$ to $6 \mathrm{GPa}$, for a Poisson's ratio of 0.25 and the yield strengths range from $19 \mathrm{MPa}$ to $25 \mathrm{MPa}$. As shown on Fig. 2, no obvious correlation between global porosities, strengths and Young's moduli values may be established. Indeed, local structural mesoheterogeneities in a sample may affect the global mechanical behavior and induce strengths and moduli fluctuations. For further details on the correlation between the mesoheterogeneity and global mechanical response, one may refer to Dautriat et al. (submitted for publication). In addition, we have performed simple compression tests on two other samples in dry condition (Fig. 2b). Unconfined Compressive Strengths (UCS) of 14 and $16 \mathrm{MPa}$, respectively, were measured. Meanwhile, larger Young's moduli respectively of 8 and 9 GPa have been estimated. These discrepancies could be related in the one hand to the fluid role, which is known to modify the mechanical behavior of rocks (Vasarhelyi, 2003) and in the other hand to the small confinement required in first set of hydromechanical experiments.
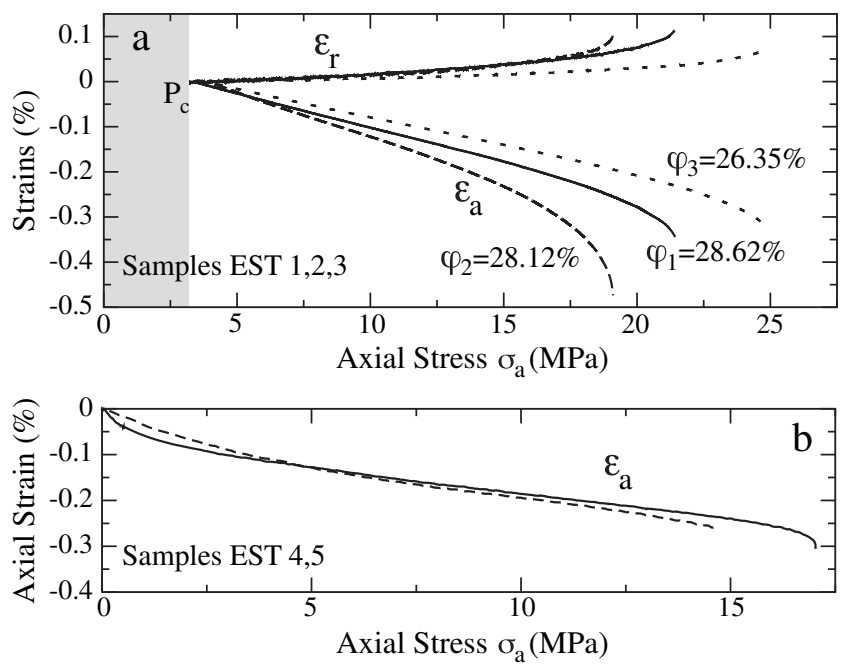

Fig. 2. (a) Stress-strain curves of three Estaillades samples, in saturated and drained conditions, from triaxial tests with low confining pressure ( $\mathrm{Pc}=3 \mathrm{MPa}$ ). (b) Stressstrain curves of two Estaillades samples, in dry condition, from simple unconfined compression tests. 
4.2. Uniaxial compression tests coupled with in-situ multi-scale observations and DIC

In the present study, "in-situ" means a combination of loading and observation set-ups allowing to continuously or stepwise (without unloading) record images from the sample surface during the compression.

\subsubsection{Uniaxial compression tests coupled to optical monitoring}

A compression loading device is used in unconfined axial compression condition, with crosshead displacement control and a recording of the applied load. As shown on Fig. 3, two digital cameras equipped with appropriate optical set-ups, described hereafter, one on each side of the sample, continuously record images at a frequency of about $0.5 \mathrm{~Hz}$. Images are recorded two surfaces covering on one side the whole sample height and on the other side a smaller zone; this set-up allows to investigate both macroscopic and mesoscopic scales. The test is performed at a constant deformation rate of $1 \mu \mathrm{m} \mathrm{s}-1$, corresponding to an axial strain rate of the order of $10-5 s-1$, up to the fracture of the sample. The overall shortening of the sample is measured by an LVDT sensor. During a test, which lasts about $10 \mathrm{~min}$, almost 250 pictures are taken at the two different scales. The optical set-up is essentially the same as the one used for clay-rocks by Bornert et al. (2010), with adapted magnifications and a better resolution camera for macro imaging. Indeed, a high resolution CCD Imperx camera $(4872 \times 3248$ pixels in 12 bits, with a pixel size of $7.4 \times 7.4 \mu \mathrm{m} 2)$ records pictures over the whole height of the sample and a CCD Spot Insight camera ( $2048 \times 2048$ pixels in 14 bits, with a pixel size of $7.4 \times 7.4 \mu \mathrm{m} 2)$ takes pictures of a $7.5 \times 7.5 \mathrm{~mm} 2$ zone, corresponding to a pixel size of $3.7 \times 3.7 \mu \mathrm{m} 2$ owing to the Mitutoyo $\times 2$ objective in use (numerical aperture of 0.055 , corresponding to a radius of Airy disk of $5.5 \mu \mathrm{m}$ ). Camera set-ups and surface lighting are also optimized to provide the widest histograms of grey levels and to improve the signal to noise ratio. Noise levels are similar in both cameras and are essentially dominated by photon shot noise (Janesick, 2001). Noise levels are below 1/100 of the full dynamic ranges of the sensors.

Accuracy of DIC measurements strongly depends on the signal to noise ratio of the images at the scale of the correlation windows. In order to increase this ratio and to provide the required grey level fluctuations at the appropriate scale, the natural contrast of the samples has been enhanced by spraying black and white paint over the white/light-grey rock surface. The droplets should ideally be of the order of a few pixels with an almost uniform size and sufficient density, as further discussed in Section 5.2. Because of the low depth of field of the optical microscope (about $90 \mu \mathrm{m}$ ), a flat surface has been machined parallel to the axis of the sample and polished. To preserve the symmetry of the test, a similar surface has been machined on the other side. Great care is taken to align at best the axes of the cameras with the normals to these flat surfaces.

Two geometries of samples have been loaded using this device. First, two experiments have been performed on flattened cylinder shaped samples, later referred to as EST_OPT_A and EST_OPT_B, respecting the conventional aspect ratio of triaxially loaded samples, i.e. 2 (100 $\mathrm{mm}$ in height and $50 \mathrm{~mm}$ in diameter). The width of the two opposite flat surfaces is about $15 \mathrm{~mm}$. In this case, the CCD Imperx camera is equipped with a $90 \mathrm{~mm}$ Apo-Componon lens and appropriate extension tubes (Unifoc 12 Macro-System), which provide an image pixel size of $\sim 22 \times 22 \mu \mathrm{m} 2$. Second, two flattened cylinder shaped samples with a smaller aspect ratio close to $1(37 \mathrm{~mm}$ in height and $36 \mathrm{~mm}$ in diameter) have been selected from cylindrical bars of $120 \mathrm{~mm}$ in height, according to their degree of heterogeneity inferred from porosity profiles obtained by X-Ray tomodensitometry as shown in Fig. 4. The first 'homogeneous' sample EST_OPT_C is characterized by a reasonably constant porosity of $25.5 \%( \pm 0.5 \%)$ along its axis. The second 'heterogeneous' sample EST_OPT_D presents a strong axial porosity gradient from $32 \%$ at the bottom to $22.5 \%$ at the top. The arrow reported on the density profile on Fig. 4 highlights the presence of an $\sim 8 \mathrm{~mm}$ long dense bioclast across the bottom of the sample EST_OPT_D, which constitutes a well-marked mesoheterogeneity in the most porous part of the sample. The two opposite and symmetric flat surfaces were this time $24 \mathrm{~mm}$ in width such that their size and shape coincide with that of the sensor of the Imperx camera. In this case, the camera is equipped with a MakroSymmar HM $120 \mathrm{~mm}$ lens and the optical magnification is set to about $\times 1$, providing a physical size of a pixel in the $16 \mathrm{M}$ pixels images of about $7.5 \times 7.5 \mu \mathrm{m} 2$. Teflon sheets have been added at the interface between sample and pistons.

\subsubsection{Uniaxial compression tests coupled to SEM imaging}

A specific miniaturized compression device (Allais et al., 1994) has been designed and adapted to fit within the chamber of the FEG-ESEM FEI Quanta600. The loading of small parallelepipedic samples ( $\sim 10 \mathrm{~mm}$ in height and $\sim 5 \mathrm{~mm}$ in width) is applied at a deformation rate of $1 \mu \mathrm{m} \mathrm{s}-1$ (corresponding to an axial strain rate of the order of $10-4 \mathrm{~s}-1$ ) in a stepwise manner. The observed side is carefully drypolished (down to grade $4000 \mathrm{SiC}$ paper) and coated with gold. As the
16 MPix CCD
Imperx Camera

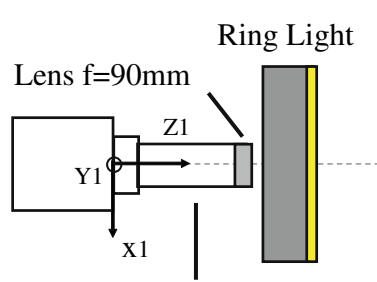

Extension tubes

Side Lightening LED

4 MPix CCD

Spot Camera

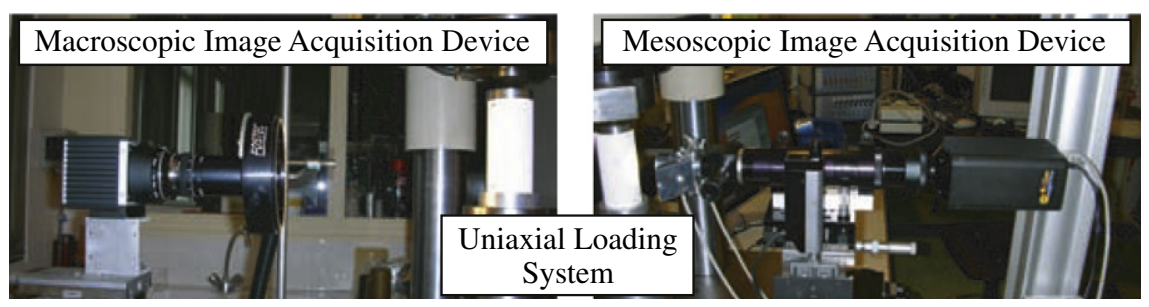

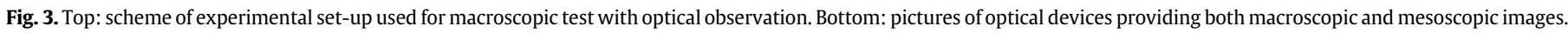


a
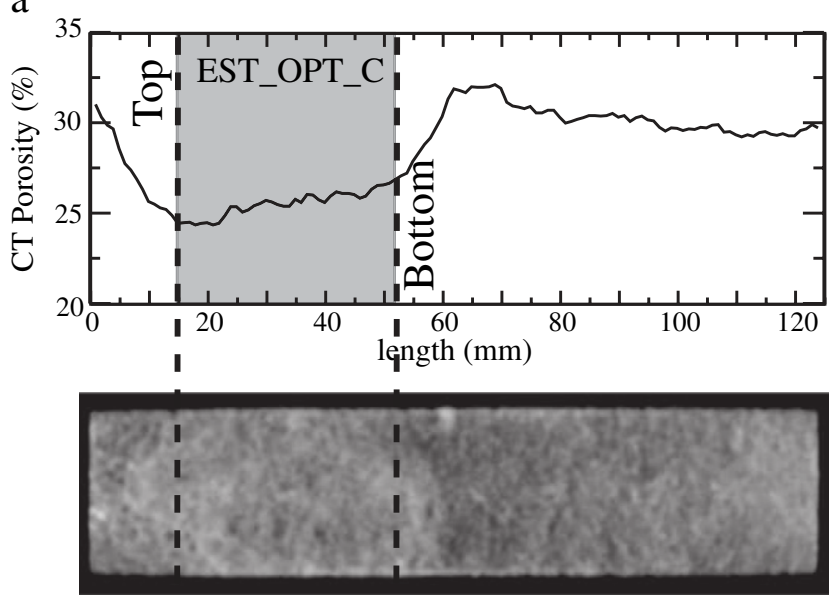

b
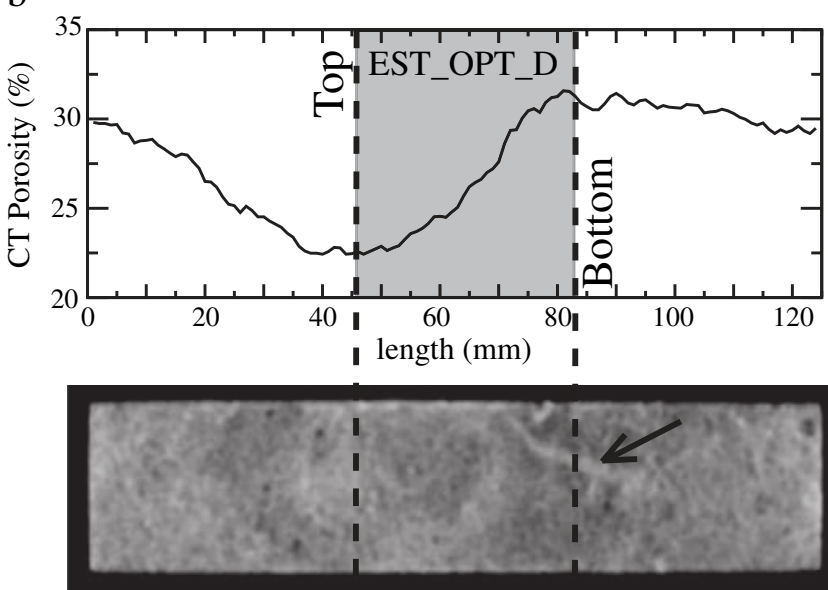

Fig. 4. Porosity profiles of two cylindrical bars of Estaillades limestone inferred from Xray tomodensitometry. The grey areas correspond to the selected small aspect ratio ( 1) samples EST_OPT_C (a) and EST_OPT_D (b), according to their respective homogeneity and heterogeneity at the mesoscale. The arrow shows a dense bioclast near the bottom of the sample EST_OPT_D.

SEM image recording times are fairly long (several minutes) and several zones were investigated on each sample, five load increments at most could be reasonably analysed during a single test (almost $12 \mathrm{~h}$ ). The images are acquired with magnifications (with respect to the image on monitor) ranging from $\times 40$ to $\times 160$ during a same test. With a magnification of $\times 40$, the high definition image $(4096 \times 3536)$ covers an area of $3.7 \times 3.2 \mathrm{~mm}^{2}$ in size for an image pixel size of $910 \times 910 \mathrm{~nm}^{2}$. This scale allows the observation of local phenomena occurring at the mesoscale. The higher magnification provides information on much finer mechanisms taking place at microscale, with an image pixel size down to $230 \times 230 \mathrm{~nm} 2$.

Secondary electron (SE) imaging sensitive to image topography provides the best contrast for DIC processing, but may also introduce some uncertainties further discussed in Section 5.3. Owing to the chemical uniformity of the carbonate sample, backscattered electrons (BSE) images did not provide an appropriate contrast. Beam size and scanning rate are also adjusted to get a good compromise between the quality of the signal to noise ratio and the problem of electrical charge accumulation. Noise levels are however larger in SEM images than in optical images, as discussed by Yang et al. (2010).

Before each image acquisition, the beam is repositioned and magnification is selected according to the position and size of the reference image; then contrast and brightness are adjusted so that the full range of 256 grey levels is covered without saturation and remains as close as possible to the grey levels of the reference image.

\section{Assessment of uncertainties linked to the DIC processing}

Since carbonate samples fail at rather low axial strain magnitudes $(<0.2 \%)$, the expected relative displacements of adjacent points remain small and often close to the limit of resolution of the DIC procedure. It is therefore of fundamental importance to quantify and control as precisely as possible, the uncertainties on image acquisitions on the one hand and on the DIC procedure on the other hand, in order to ensure a successful application of the technique.

\subsection{Various sources of errors on displacement measurements}

DIC-based measurements can be tainted by errors related to both imperfections of image acquisition and DIC processing.

A first source of errors is the noise in the image. In the case of optical imaging, the noise levels are strongly dependent on the characteristics of the sensor. Here, the noise is essentially controlled by the photon shot noise. Conversely, SEM images are inherently noisier, due the image reconstruction technique. To improve the image quality, the size of the electron beam and the raster like scanning rate must be appropriately selected. For instance, a large spot size and a slow scanning rate (either $30 \mu$ or $15 \mu \mathrm{s}$, corresponding respectively to acquisition times of 7 and $3.5 \mathrm{~min}$ ) allow a good averaging of the signal transmitted to the secondary electron sensor. Image noise has to be compared to the actual contrast in the correlation domains, because DIC random error is more or less proportional to the ratio between noise and available local contrast within each domain (Roux and Hild, 2006). This ratio could be decreased by an enhancement of the local contrast. Black and/or white paint microdroplets (size ranging from 10 to $100 \mu \mathrm{m}$ ) have been sprayed on samples subjected to macroscopic test. For SEM in Secondary Electron mode, which is sensitive to the roughness of the surface, no additional marking of the surface is needed, since the microheterogeneity ensures already a sufficient contrast for DIC. Another way to decrease the noise to contrast ratio consists in enlarging the size of correlation domains, at the cost of a reduced spatial resolution of the displacement field.

A second source of errors lies in the DIC algorithm itself. Shape function mismatch errors, discussed by Schreier and Sutton (2002) and more precisely by Bornert et al. (2009), are likely to be limited in our case, because of the low level of strains (a strain of $0.1 \%$ induces 0.03 maximum relative pixel motion in a 30 pixels wide correlation window). A more critical source of error at low strains is linked to the interpolation of the grey levels in the correlation domains, which is performed in order to get the displacement with sub-pixel accuracy (Schreier et al., 2000; Schreier and Sutton, 2002; Bornert et al., 2009). This error, so-called "systematic error", corresponds to an under or over estimation of the actual displacement, which is correlated to the fractional part of the displacement expressed in pixel. It can be strongly dependent on the interpolation algorithm (Schreier et al., 2000) and on the available image contrast, but the detailed dependence is not yet well understood; according to the estimation of the uncertainties, bi-linear and bi-cubic interpolations providing roughly the same amplitude of the systematic error, the former was used in this study. At very low strains, the practical consequence is the occurrence of "moiré-like" fringes on the deformation map, inducing a large error on the local deformation calculation and thus masking locally the actual deformation field. This error may be quantified by an S-shape curve, yielding the intensity of the error as a function of the fractional part of the displacement.

The third kind of errors in 2D-DIC investigations is linked to geometric errors in the reference or deformed images. SEM images are more prone to such errors, due to instabilities of the scanning system (fluctuation in the beam deflections, imperfection of scan generator, beam drift...). Optical images are less sensitive to these errors since CCD sensors have a fixed and well defined geometry. Since 
displacements are small, the distortions are similar in the reference and deformed images and cancelled out of the DIC analysis.

The last source of errors is linked to a potential non-controlled outof-plane rigid body motion of the sample with respect to the observation device. For optical imaging, global sample motion sample within the depth of field results in a slight modification of the magnification, inducing uniform isotropic apparent strain. This effect may be easily quantified by moving the sample by a known number of pixels toward the lens to generate a dilation of the image. This rigid body motion may also be a rotation about an axis normal to the optical one, inducing a slight gradient of magnification on the observation surface and apparent in-plane deformation.

In this study, the errors are estimated by evaluating by DIC the apparent displacement and strain field on several images acquired before the loading, following the procedure of Doumalin (2000). First, two images are acquired successively in the same conditions, i.e. without camera or microscope plate motions for optical and SEM imaging respectively. The apparent deformation computed from the correlation of those two images reflects the noise induced by the fluctuation of the conditions of the image acquisition. Then, we perform DIC computation between a reference image and an image acquired after performing an arbitrary rigid body translation in the $\mathrm{X}$ and $\mathrm{Y}$ directions. This later procedure allows two kinds of error estimation: (1) the standard deviation and the maximum error on the displacement calculation (in the $\mathrm{X}$ and $\mathrm{Y}$ directions), with respect to the average translation; (2) the standard deviation of the difference between the calculated displacement and the displacement corresponding to the homogeneous transformation equal to the mean global deformation gradient evaluated from a contour integration on the whole area of interest. Finally, one may quantify the systematic error by DIC procedure on images acquired after a motion of the sample orthogonal to the observation plane ( $Z$ direction). This imposed magnification variation is equivalent to an isotropic global deformation of few pixels in both directions $\mathrm{X}$ and $\mathrm{Y}$, and allows the occurrence of "moiré-like" bands related to the fractional part of the displacement. The systematic error is then expressed as the difference between the evaluated and theoretical displacements, associated with the global camera motion. Additional information on this procedure of systematic-error estimation is reported in Yang et al., 2010.

The error on the displacement field propagates to the calculation of the transformation gradient $\underline{F}$. However a suitable choice of the grid size and of the number of neighboring points for displacement integration can minimize this effect. For example in the given direction $\mathrm{X}$, the standard deviation of the local transformation gradient $\sigma\left(F_{x x}\right)$ is expressed as:

$\sigma\left(F_{x x}\right)=\frac{\sqrt{3}}{4} \frac{\sigma_{x}}{a}$

with $a$ the grid step and $\sigma_{x}$ the standard deviation of the error on the $x$ component of the displacement (Allais et al., 1994). The standard deviation on the global deformation calculation of a grid composed of $\mathrm{N}$ lines and a spacing $\mathrm{L}$ between the first and the last column becomes:

$\sigma\left(F_{x x}\right)=\sqrt{\frac{2}{N}} \frac{\sigma_{x}}{L}$

The uncertainties on the transformation gradient are also quite sensitive to the magnification fluctuation $\Delta m$ (i.e., the global or nonuniform sample motions outside the observation plane). The corresponding deviation can be expressed as (Doumalin, 2000):

$F_{x x}=\left(1+\frac{\Delta m}{m}\right) \frac{\partial x}{\partial X}$ with $X$ and $x$ the real coordinates of the point in the reference and deformed configurations respectively.

In the following two sub-sections, we present the results obtained with the best configurations for both image acquisition and DIC parameters obtained from several tested configurations in order to reduce the noise levels. The given DIC parameters are the ones used to interpret our experimental results in terms of global and local deformations.

\subsection{Uncertainties on the DIC processing of optical images}

For both DIC processings, the correlation domain size and the gage length for local strain measurement are fixed to 50 and 130 pixels respectively (grid step a being 65 pixels). To the first order, errors inferred from the rigid body motion procedure are quite small in both macroscopic and mesoscopic image processings. In the case of the macroscopic image, the deposit of a speckle pattern induced a strong reduction of the uncertainties. For example, the uncertainty initially of $\sim 0.032$ pixel on local displacements evaluated from the analysis of a rigid body translation in both directions is reduced by 2 after the speckle pattern application. The impact of the speckle pattern is even much more pronounced for the mesoscopic image processing; the uncertainties are 2 and 10 times smaller in the $\mathrm{X}$ and $\mathrm{Y}$ directions respectively after the speckle deposition. Indeed, paint droplets, whose size and spacing range from 1 to $100 \mu \mathrm{m}$, strongly enhance the local natural contrast at the mesoscale, which was definitively too low for the required accuracy. At the macroscale, the macroporosity provides a good contrast at the right scale, and the addition of microdroplets is less beneficial. Table 1 summarizes the evaluations of experimental errors deduced from the in-plane rigid body motion procedures described in the previous section for both macroscopic and mesoscopic images, for the sample with paint droplets.

From the values reported in Table 1 and according to the expression (3), the uncertainties on the calculated in-plane local strain components at the macroscopic and mesoscopic scales are respectively $\sim 6.6 .10-5$ and $\sim 6.6 .10-4$. In Section 6 , several sequences of deformation maps at different scales will be presented to discuss the deformation localization and the damage progression. Their interpretation was done taking into account the uncertainty levels calculated and reported here.

The results from the third procedure aiming at quantifying the systematic and random errors separately for macroscopic DIC are presented in Fig. 5, in the form of apparent deformation maps and Sshaped curves, for both samples with and without speckle paint. Here,

\section{Table 1}

Estimation of the uncertainties on the displacement field calculation for images acquired at both macroscopic and mesoscopic scales. The step of the grid is set to 65 pixels, corresponding to a strain gage of 130 pixels in both configurations. The grey levels are interpolated by a bi-linear function.

\begin{tabular}{|c|c|c|c|c|}
\hline & \multicolumn{2}{|c|}{$\begin{array}{l}\text { Macroscopic images } \\
\text { Imperx camera }\end{array}$} & \multicolumn{2}{|c|}{$\begin{array}{l}\text { Mesoscopic images } \\
\text { SPOT camera }\end{array}$} \\
\hline & $\begin{array}{l}\text { Without } \\
\text { translation }\end{array}$ & $\begin{array}{l}\text { Translation } \\
\text { in X dir. }\end{array}$ & $\begin{array}{l}\text { Without } \\
\text { translation }\end{array}$ & $\begin{array}{l}\text { Translation } \\
\text { in X dir. }\end{array}$ \\
\hline $\begin{array}{l}\text { Pure translation } \\
\text { Standard deviation } \\
\left(\mathrm{u}_{\mathrm{X}}, \mathrm{u}_{\mathrm{Y}}\right) \\
\text { Maximum deviation }\end{array}$ & $\begin{array}{l}(0.019 ; \\
0.015) \\
(0.054 ; \\
0.069)\end{array}$ & $\begin{array}{l}(0.018 \\
0.019) \\
(0.058 \\
0.12)\end{array}$ & $\begin{array}{l}(0.038 ; \\
0.034) \\
(0.90 ; \\
0.82)\end{array}$ & $\begin{array}{l}(0.17 ; \\
0.22) \\
(0.93 ; \\
0.93)\end{array}$ \\
\hline $\begin{array}{l}\text { Homogenous def. } \\
\text { Standard deviation } \\
\quad\left(\mathrm{u}_{\mathrm{X}}, \mathrm{u}_{\mathrm{Y}}\right)\end{array}$ & $\begin{array}{l}(0.020 \\
0.016)\end{array}$ & $\begin{array}{l}(0.018 \\
0.010)\end{array}$ & $\begin{array}{l}(0.061 ; \\
0.21)\end{array}$ & $\begin{array}{l}(0.18 \\
0.22)\end{array}$ \\
\hline $\begin{array}{l}\text { Global def. } \\
\varepsilon_{11} \\
\varepsilon_{22} \\
\varepsilon_{12}\end{array}$ & $\begin{array}{l}-1.81 .10^{-5} \\
-2.32 .10^{-6} \\
-3.22 .10^{-6}\end{array}$ & $\begin{array}{l}-5.23 .10^{-5} \\
-5.28 .10^{-6} \\
-3.54 .10^{-6}\end{array}$ & $\begin{array}{l}9.34 .10^{-6} \\
-1.73 .10^{-5} \\
-4.00 .10^{-5}\end{array}$ & $\begin{array}{l}-5.96 .10^{-5} \\
1.68 .10^{-5} \\
-8.52 .10^{-5}\end{array}$ \\
\hline
\end{tabular}



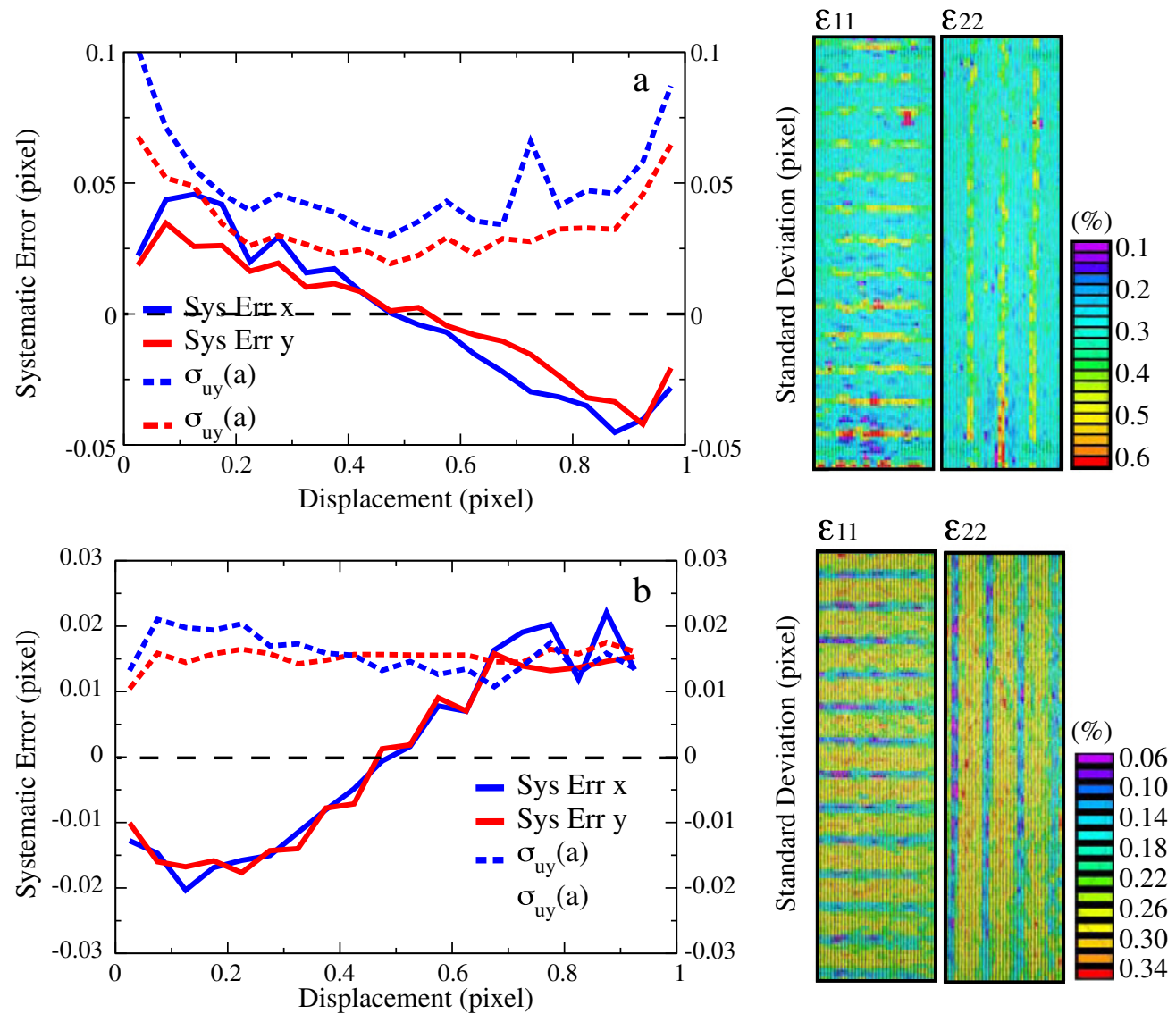

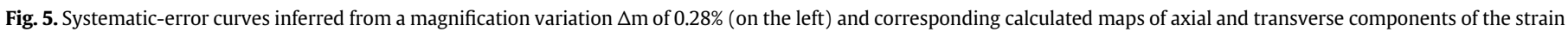

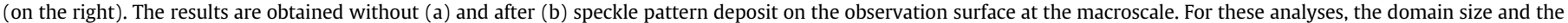
strain gage length are set respectively to 50 and 130 pixels.

the camera is moved $1 \mathrm{~mm}$ away from the sample between the two image acquisitions, corresponding to a magnification variation $\Delta m$ of $0.28 \%$. The number of periodic moiré-like bands (13 in the $\mathrm{X}$ direction and 4 in the $Y$ direction) is consistent with the higher amplitude of the global relative displacement equals to 13 pixels in the $\mathrm{X}$ direction. Again, the speckle pattern tends to significantly reduce both standard deviation and systematic-error amplitudes. As reported on Fig. 5, the maximum amplitude of the systematic-error changes from $\sim 0.045$ pixel to $\sim 0.02$ pixel before and after speckle pattern application. Furthermore, consistently with the results of Table 1, the random error decreased from [0.025-0.1] to $\sim 0.015$ pixel. For mesoscopic scale imaging, the amplitude of the systematic error is however difficult to estimate. Indeed, the small optical depth of field of the device induces a strong image disturbance even for a very small

Table 2

Estimation of the uncertainties on the displacement field calculation for images acquired by SEM. The step of the grid is set to 30 pixels, corresponding to a strain gage of 60 pixels in both configurations. The grey levels are interpolated by a bi-linear function.

\begin{tabular}{lll}
\hline & Without translation & Translation in $\mathrm{X}$ and $\mathrm{Y}$ dir. \\
\hline $\begin{array}{l}\text { Pure translation } \\
\text { Standard deviation }\left(\mathrm{u}_{\mathrm{X}}, \mathrm{u}_{\mathrm{Y}}\right)\end{array}$ & $(0.059 ; 0.051)$ & $(0.15 ; 0.18)$ \\
Maximum deviation & $(1.27 ; 0.58)$ & $(1.65 ; 2.81)$ \\
& & \\
Homogenous def. & & \\
Standard deviation $\left(\mathrm{u}_{\mathrm{X}}, \mathrm{u}_{\mathrm{Y}}\right)$ & $(0.075 ; 0.064)$ & $(0.19 ; 0.18)$ \\
Global def. & & \\
$\quad \varepsilon_{11}$ & $-5.33 .10^{-5}$ & $-2.69 .10^{-5}$ \\
$\varepsilon_{22}$ & $1.57 .10^{-5}$ & $-4.33 .10^{-5}$ \\
$\varepsilon_{12}$ & $1.57 .10^{-5}$ & $-5.08 .10^{-6}$ \\
\hline
\end{tabular}

magnification fluctuation. To limit the error due to out-of-plane deformations, the focusing is controlled and adjusted continuously during the experiment. Errors induced by magnification fluctuations are thus limited at the mesoscale.

\subsection{Uncertainties on the DIC processing of SEM images}

The uncertainties on the DIC evaluation of displacement fields deduced from SEM images have been determined for a magnification $\times 80$ and are reported in Table 2 . The image correlation parameters are a domain size of 30 pixels and a local gage length of 60 pixels. Fig. 6 is a map of the equivalent deformation measured between two images

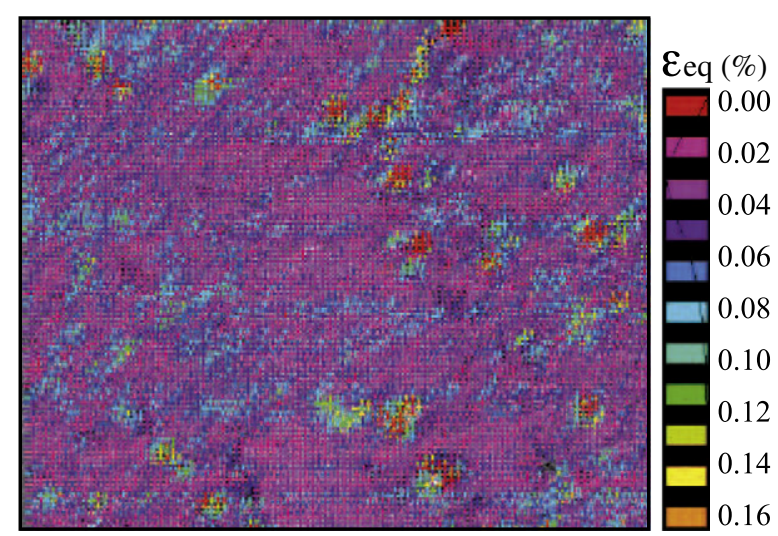

Fig. 6. Equivalent deformation map from DIC processing of two images acquired without rigid body translation, corresponding to the minimum level of uncertainty. 


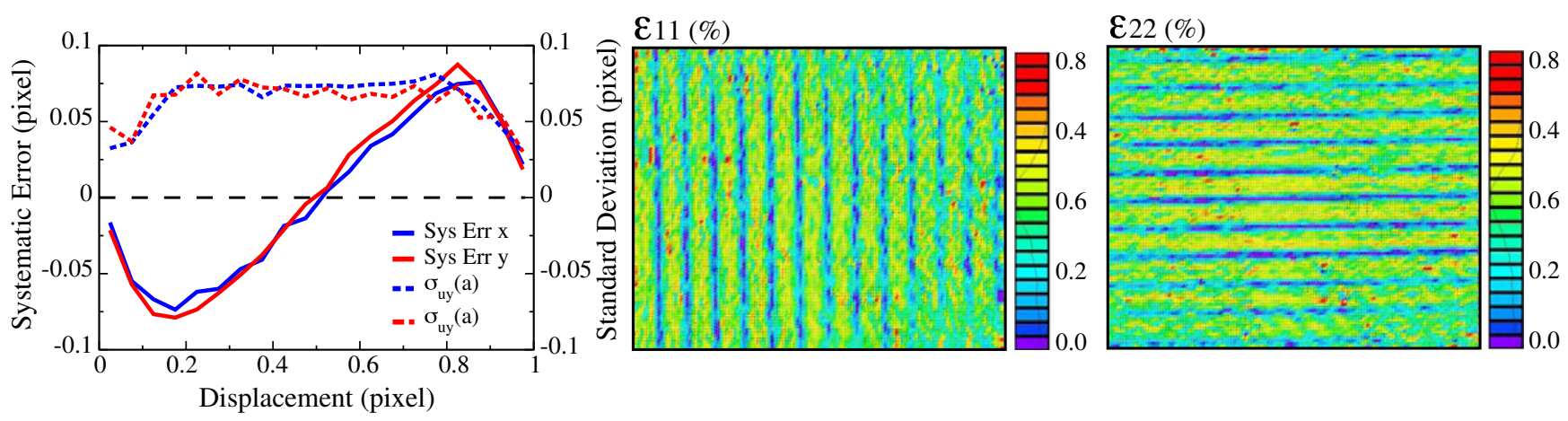

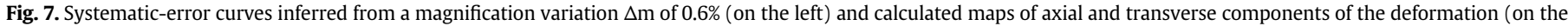
right), obtained on SEM images at $\times 80$ magnification. For these analyses, the correlation domain size and the strain gage length are set respectively to 30 and 60 pixels.

acquired without any sample motion and thus illustrates the minimum level of error that can be expected. We note that error magnitudes are correlated with topography and more specifically are larger in areas where suitable evacuation of electronic charges is limited. These areas correspond to discontinuities of the surface metallization in the larger pores. The most affected areas could be detected and manually removed from the DIC computation. All other uncertainties inherent to the SEM are rather limited (about 0.13 pixel). This result contrasts sharply with previous studies, (Racine, 2005; Héripré et al., 2007), which report 3 to 5 times higher error levels. This improvement is due to the more accurate beam control system, which equips the new generation SEM used in this study, as well as to more limited levels of noise.

From the values presented in Table 2 and according to expression (3), the uncertainties on the calculated in-plane local deformation components (inherent to a slight error of beam repositioning between two image acquisitions and image noise, and corresponding to in-plane translations) is $\sim 1.2 .10-3$. In the Section 6.4 , we will present deformation maps highlighting strong localization events, for which the deformation amplitudes are much higher than the estimated uncertainty levels.

The systematic error on the displacement measurement has been quantified for a magnification variation $\Delta m$ of $0.6 \%$, without beam focusing between the two image acquisitions. The consequent occurrence of moiré-like bands is clearly observed in Fig. 7. The maximum amplitude of the systematic error is equal to 0.075 pixel for a random error close to the one calculated by a rigid body translation. Compared to the macroscopic image, the larger value of the systematic error is mostly related to the nature of the local contrast provided by the microporous aggregates. On the one hand, the roughness of the aggregate surface creates a conveniently wide grey level distribution, but on the other hand, it also results in sharp local fluctuations with very short variation lengths, which are hard to reproduce accurately by interpolation algorithms. To limit this effect, a small unfocusing is applied for each image, in order to slightly smooth the local contrast in the microporous aggregates.

\section{Experimental results}

\subsection{Macroscopic DIC applied on large samples}

Fig. 8 presents the results of both axial $(\varepsilon 11)$ and transverse $(\varepsilon 22)$ components of the global deformation inferred from macroscopic and mesoscopic DIC computations of images acquired during the compression of the sample EST_OPT_A as a function of the applied stress. In addition, conventional axial strain measurement by LVDT is also reported. We show that the results of DIC measurements at the macroscopic and the mesoscopic scales are consistent. The latter are noisier, owing to sensitivity to vibrations and optical instability of the mesoscopic observation device, as well as to the lower signal to noise ratio of the mesoscopic images in which the contrast is less appropriate and the presence of more pronounced systematic errors. The DIC analyses show that the brittle failure of the sample is obtained for an axial strain of $\varepsilon a \sim 0.11 \%$ at a peak stress of $\sim 16 \mathrm{MPa}$ (consistent with previous results, Fig. 2b), whereas the LVDT measurement provides a higher value of $\varepsilon a \sim 0.37 \%$, due to both sample positioning at the beginning of the experiment and compliance of the testing device. The DIC macroscopic measurement yields a Young's modulus $E \sim 13.9 \mathrm{GPa}$ and a Poisson's ratio $\nu \sim 0.21$, whereas the LVDT measurement provides a much lower value of $E \sim 5.8 \mathrm{GPa}$.

Fig. 9 presents the deformation maps calculated at four selected levels of loading, indicated in Fig. 8. The colour is associated with the intensity of the equivalent deformation eeq. During the initial loading phase (macroscopic stress $\sigma<5 \mathrm{MPa}$ ), the strain field remains homogeneous. At an early stage of the roughly linear part of the macroscopic stress-strain response, a lens-shaped zone of higher strain level appears in the upper part of the sample and is well identified on the deformation map when the axial stress reaches $\sigma \sim 7.5 \mathrm{MPa}$ (Fig. 9 - image A). This lens further develops as shown on the map at $\sigma \sim 12.5 \mathrm{MPa}$ (Fig. 9 - image B) and generates a deformation gradient along the sample at $\sigma \sim 15 \mathrm{Mpa}$ (Fig. 9 - image C). At this stage, local equivalent strains (relative to a gage length of 130 pixels, i.e. $2.8 \mathrm{~mm}$ ) may differ by one order of magnitude. At the same time, at the mesoscopic scale in a selected area within the upper part of the

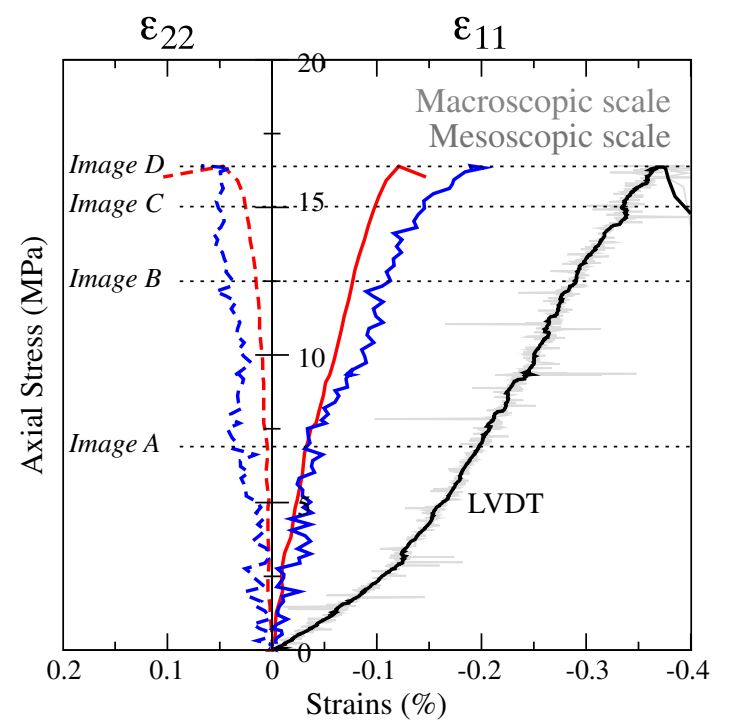

Fig. 8. Stress-strain curves with different strain estimations: axial LVDT measurements and computed axial and transverse components of deformation from DIC at both macroscopic and mesoscopic scales. The referred images A to D correspond to the stress levels, at which equivalent deformation maps, presented in Fig 9, are obtained. 

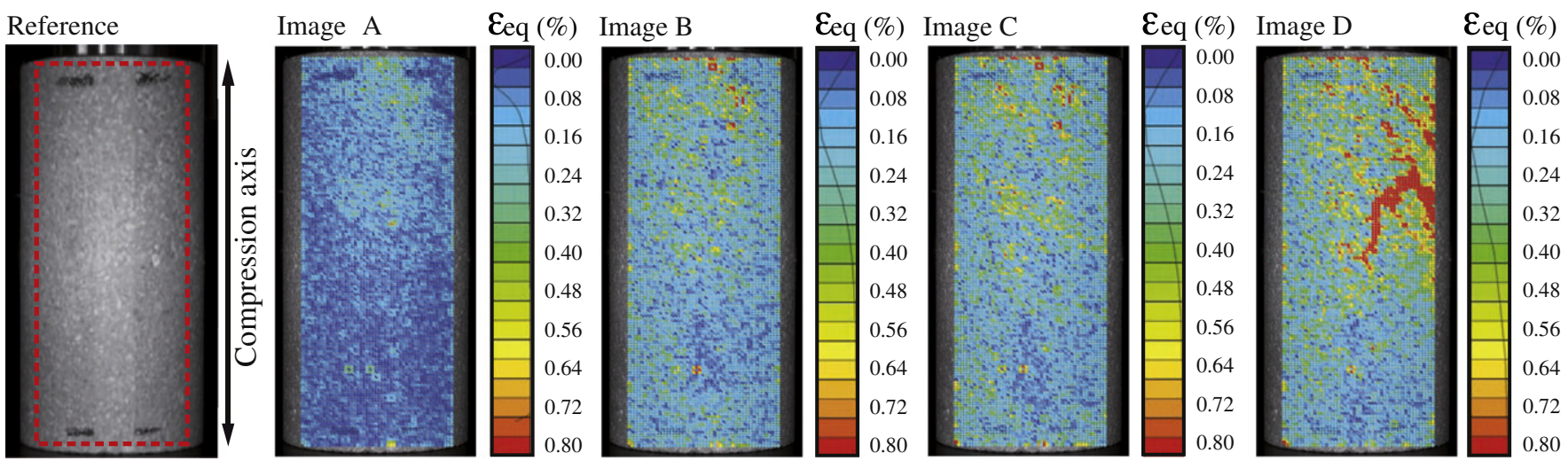

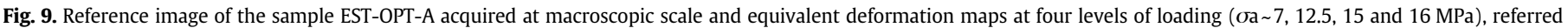
images A to D on Fig. 8. Compression acts vertically. The correlation domain size and the strain gage length are set respectively to 50 and 130 pixels.
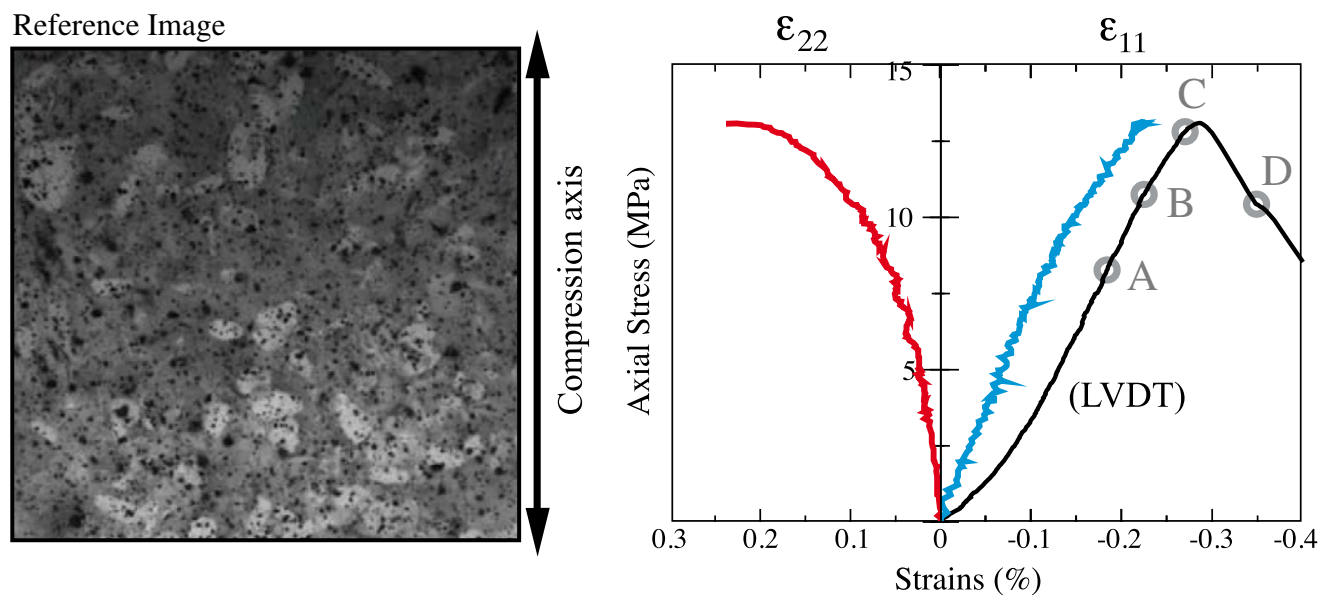

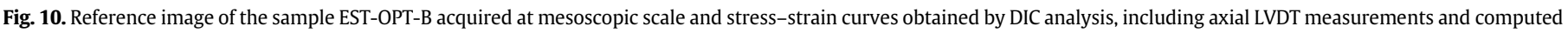

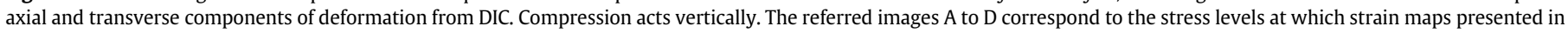
Fig. 11 are obtained.
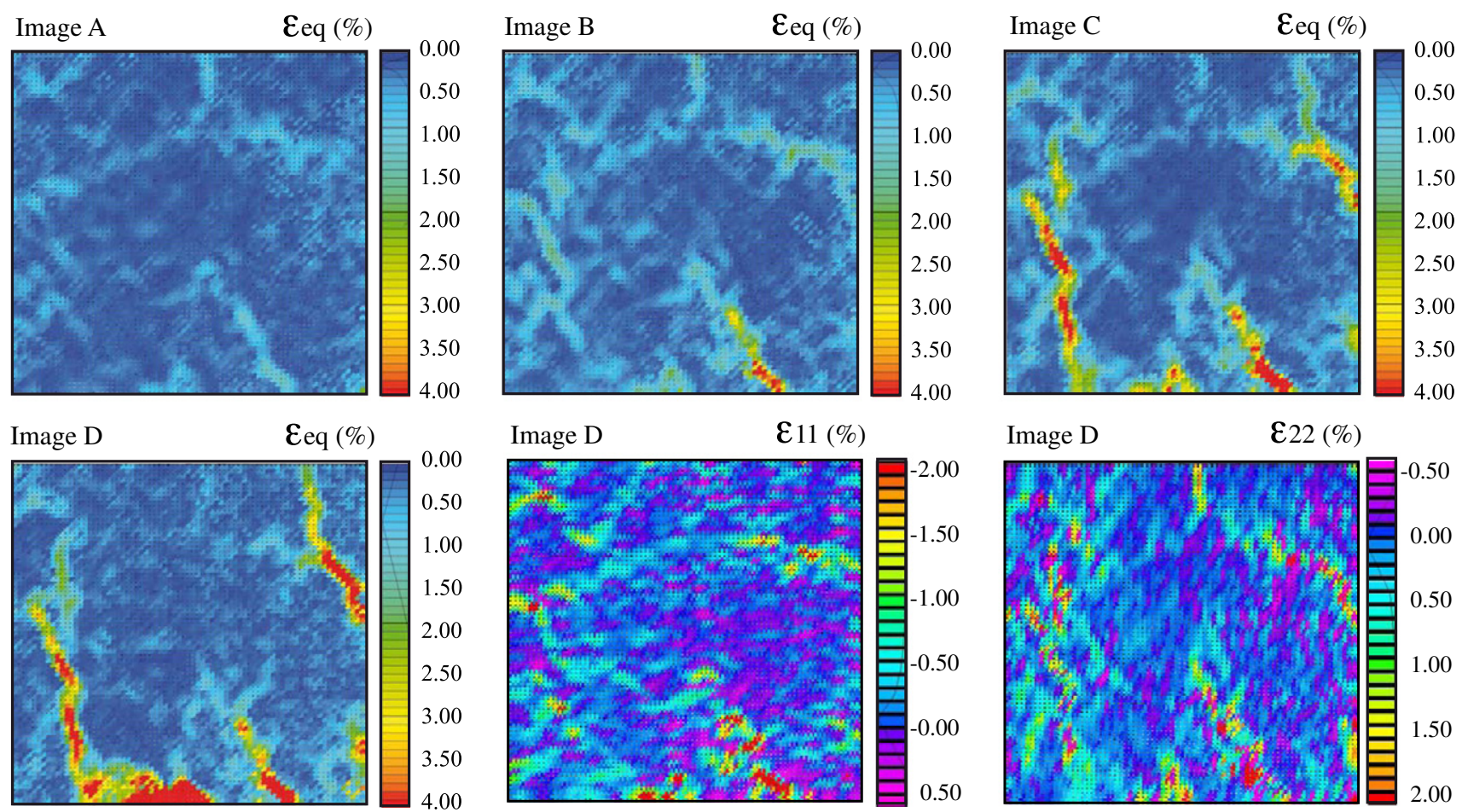

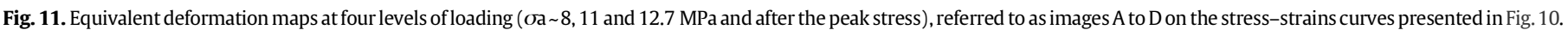
Additionally, maps of both axial and transverse strain components are reported for image $\mathrm{D}$. The correlation domain size and the gage length are set respectively to 50 and 130 pixels. 
sample, the equivalent strain is about three times larger than in the lower part. Just before the peak stress close to $\sigma \sim 16 \mathrm{MPa}$ (Fig. $9-$ Image D), at the onset of dilatancy, a strong deformation localization is revealed by DIC, and prefigures the zone of location of the macroscopic fracture propagation. The structural analysis along the observation surface provides consistent information to interpret the strain maps. The surface shows a higher concentration of clusters of dense aggregates in the central zone of the sample, where the fracture propagates, whereas in the lower part, a more homogeneous repartition of dense and microporous aggregates is observed. As it will be discussed in Section 6.3, a characterization of the initial threedimensional heterogeneity remains necessary to better understand its impact on the heterogeneity of the strain field and its evolution.

\subsection{Mesoscopic DIC applied on large samples}

At the mesoscopic scale, the operator has to select arbitrarily an observation area. In the present case, the area selected for the sample EST_OPT_A did not provide any evidence of localization during the sample loading. We present therefore only the observation at the mesoscopic scale for the sample EST_OPT_B. The selected image is characterized in lower and upper parts by different concentrations of dense aggregates, while the central part is composed almost exclusively of microporous aggregates (Fig. 10). Fig. 11 presents the macroscopic loading curve of the second sample EST_OPT_B and five images labeled A to $\mathrm{D}$ showing the deformation maps calculated at the four respective levels of loading shown on the loading curve; in addition on the last image $\mathrm{D}$, the other in-plane components have been reported. In agreement with the macroscopic analysis, the deformation field seems to be homogeneous during the initial loading, up to $\sigma \sim 5 \mathrm{MPa}$ and despite the structural heterogeneities, the DIC analysis at mesoscale does not reveal any localization in the studied region. However, for a stress level of about $\sigma \sim 8 \mathrm{MPa}$, different events of localization appear successively in weaker zones, corresponding to the opening of interaggregate porosity (mesoporosity) normal to the loading direction and the relative motion of surrounding aggregates (Fig. 11 - images A and B). These microcracks propagate along the direction of loading, around both denser and microporous aggregates gathered at the center of the zone, and reach a maximum density just before the peak stress at $\sigma \sim 13 \mathrm{MPa}$ (Fig. 11 - image C). A strong relaxation associated with the coalescence of the microcracks through the denser aggregates follows the macroscopic failure (Fig. 11 - image D).

Our analysis shows that the ultimate macroscopic fracture propagation results from the gradual opening of microcracks and their coalescence at the mesoscale. The occurrence and development of these cracks are controlled by the repartition of dense and loose aggregates. The dilatant or shear character of the localization depends on the orientation of the microcracks, parallel or transverse to the axial stress direction respectively. This aspect will be further investigated at a finer scale on both in-plane components using SEM images processing.

\subsection{Additional macroscopic DIC results on smaller samples}

Fig. 12a presents the results of DIC applied on the homogeneous sample EST_OPT_C, with a nearly constant porosity of $25.5 \%$ and maps of equivalent deformation, referred to on the strain-stress curve by points $A$ to $D$ and after failure (E). The slope of stress-strain curve calculated through the DIC is large and corresponds to a much higher value of the Young's modulus $E \sim 24.6 \mathrm{GPa}$ for this sample compared to the sample EST_OPT_A. The non linearity of the overall stress-strain curve at low stress ( $\varepsilon 11$ and $\varepsilon 22$ are under and over estimated respectively at the beginning) might be explained by a slight global out-of-plane motion of the sample, which induces small optical magnification variations. The intensity of this global out-of-plane motion is however limited; while it does not significantly affect the
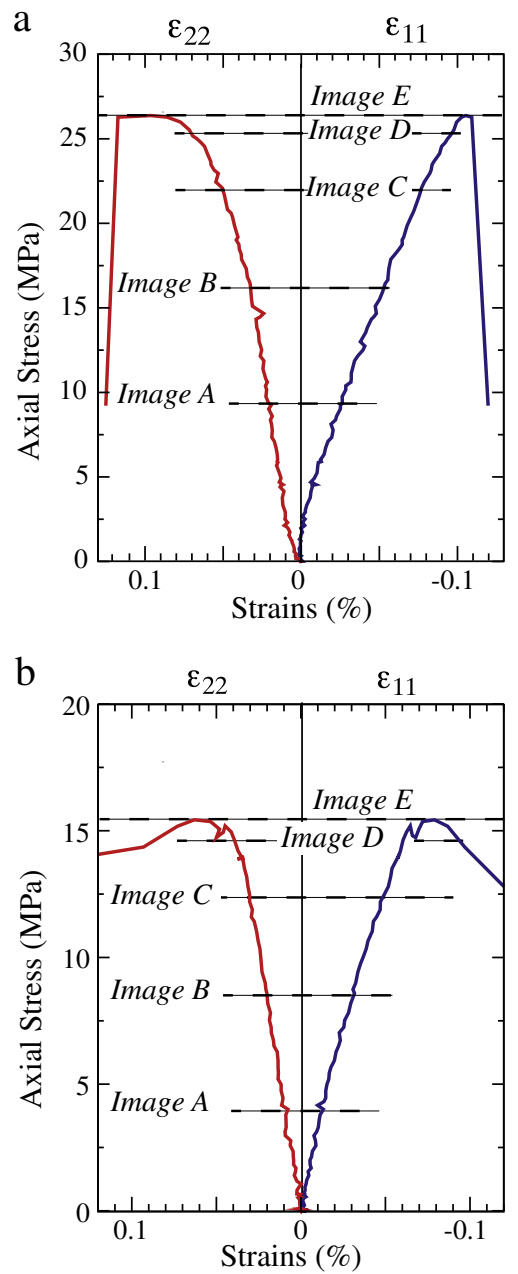

Fig. 12. Stress-strains curves of both axial and transverse components of strains from DIC processing on samples EST_OPT_C (a) and EST_OPT_D (b) at macroscopic scale. The referred images $A$ to $E$ correspond to the stress levels, at which strain maps presented in Fig. 13 for the sample EST_OPT_C and Fig. 14 for the sample EST_OPT_D are obtained.

local strain heterogeneity in the deformation maps, it has a significant impact on the apparent elastic moduli values. In future works, the procedure developed by Yang et al. (2010) could be used to correct the effects of apparent global strain induced by this out-of-plane motion.

The brittle failure of EST_OPT_C sample is obtained for $\varepsilon a \sim 0.11 \%$ (also affected by the variations of the magnification during the test) and at a peak stress of $\sigma \sim 26.4 \mathrm{MPa}$, which is similarly much higher than for the larger samples. As shown in Fig. 13, the homogeneity of the sample results in a homogeneous strain field during the loading, without localized deformation. From stress level $\sigma \sim 22 \mathrm{MPa}$, the central part of the observation surface shows the nucleation and the opening of microcracks. From this stage, the microcracks tend to progressively link up, to finally induce a failure propagated at $30^{\circ}$ of the axial stress direction, as expected from theory (Paterson and Wong, 2005).

The results obtained on the heterogeneous sample EST_OPT_D are presented in Fig. 12b. This sample is less stiff and less resistant, the brittle failure being reached earlier, at $\varepsilon a \sim 0.08 \%$ for an axial stress of $\sim 15.4 \mathrm{MPa}$. This heterogeneous sample exhibits a lower value of the apparent Young's modulus E 21.9 GPa than the homogeneous EST_OPT_C. The much higher value of strength of both samples EST_OPT_C and EST_OPT_D compared to the previous studied macroscopic samples, could be related to the lower aspect ratio and larger flat surfaces of these samples. Indeed, Hoek and Brown (1980) 


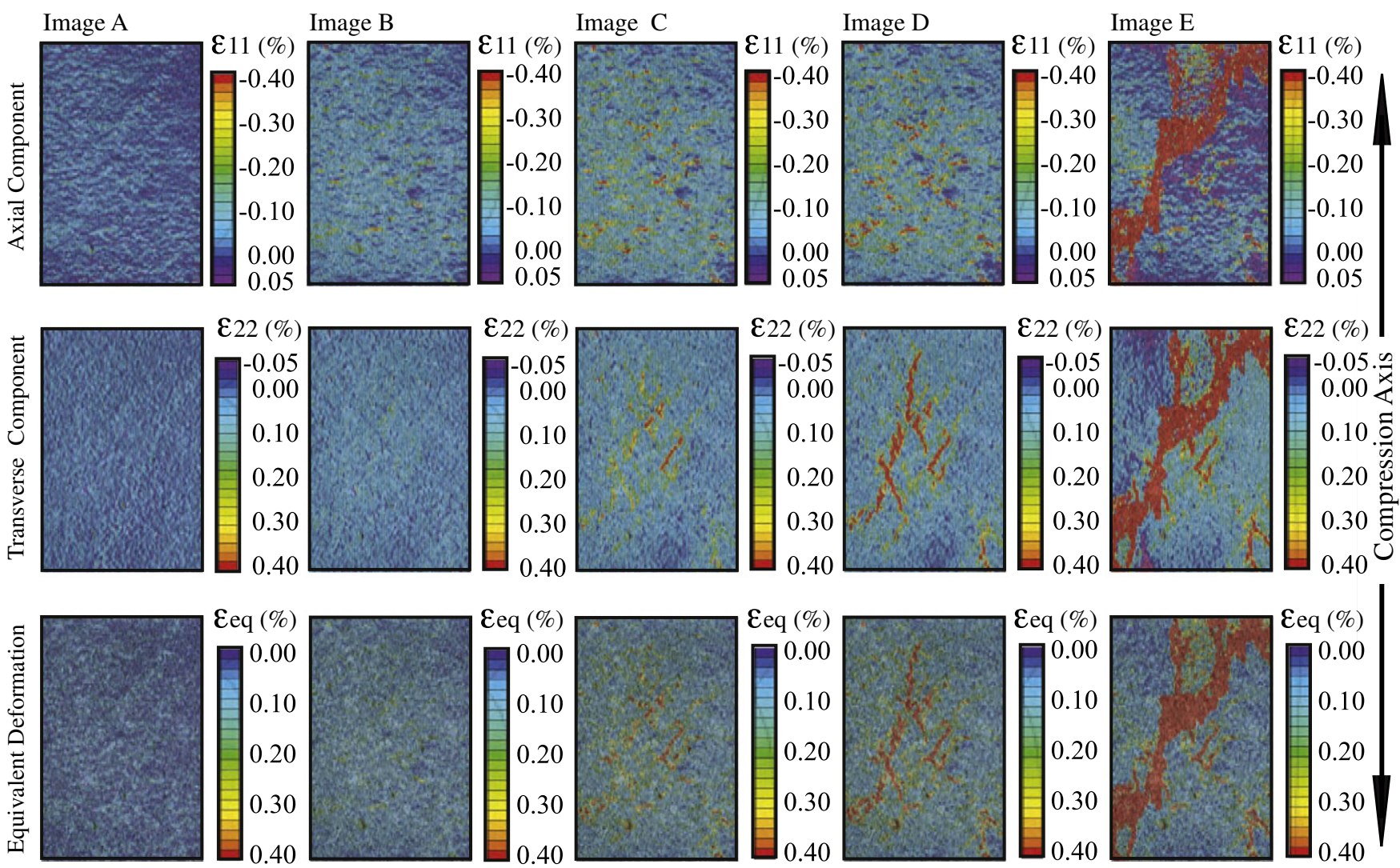

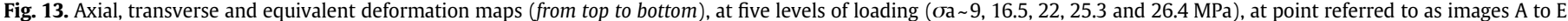

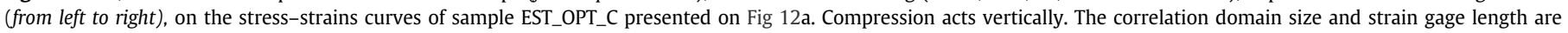
respectively set to 50 and 130 pixels.

Image A
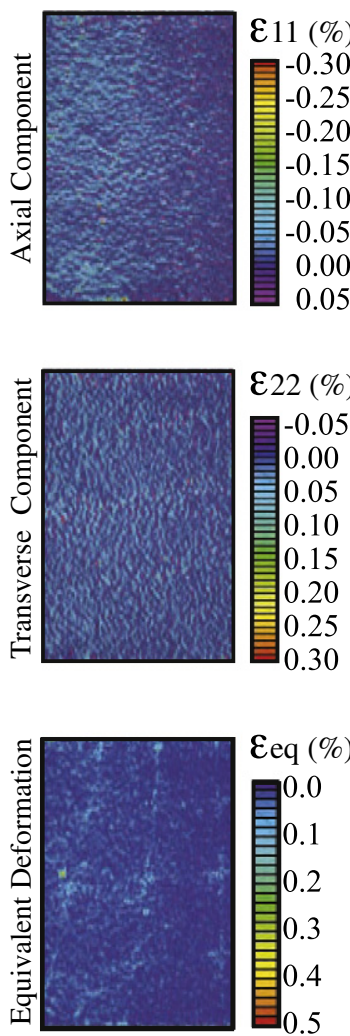

Image B
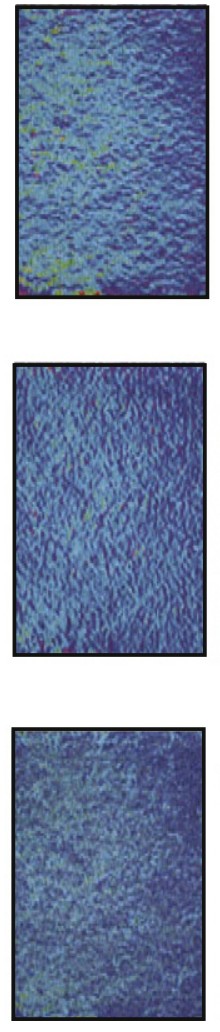

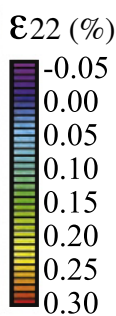

Image $\mathrm{C}$

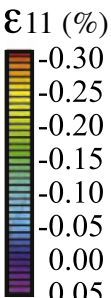

0.05

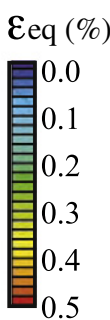

Image D

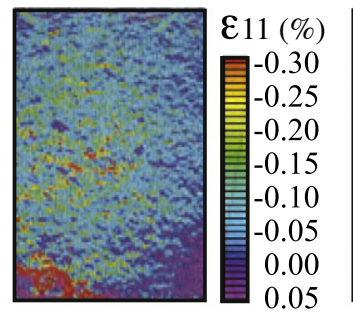

Image $\mathrm{E}$
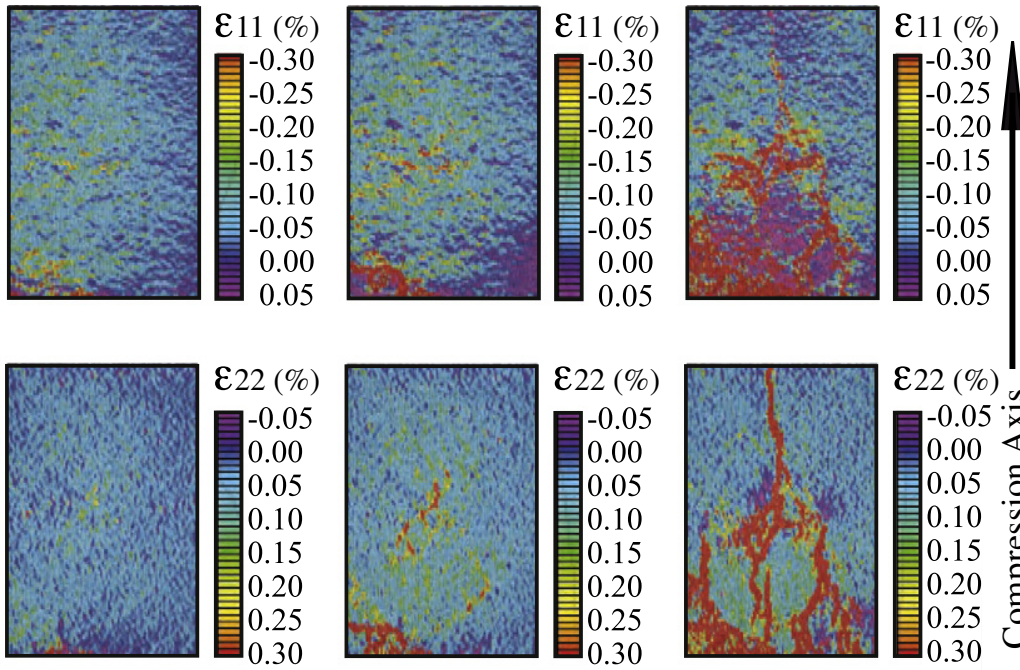

$\varepsilon_{22}(\%)$
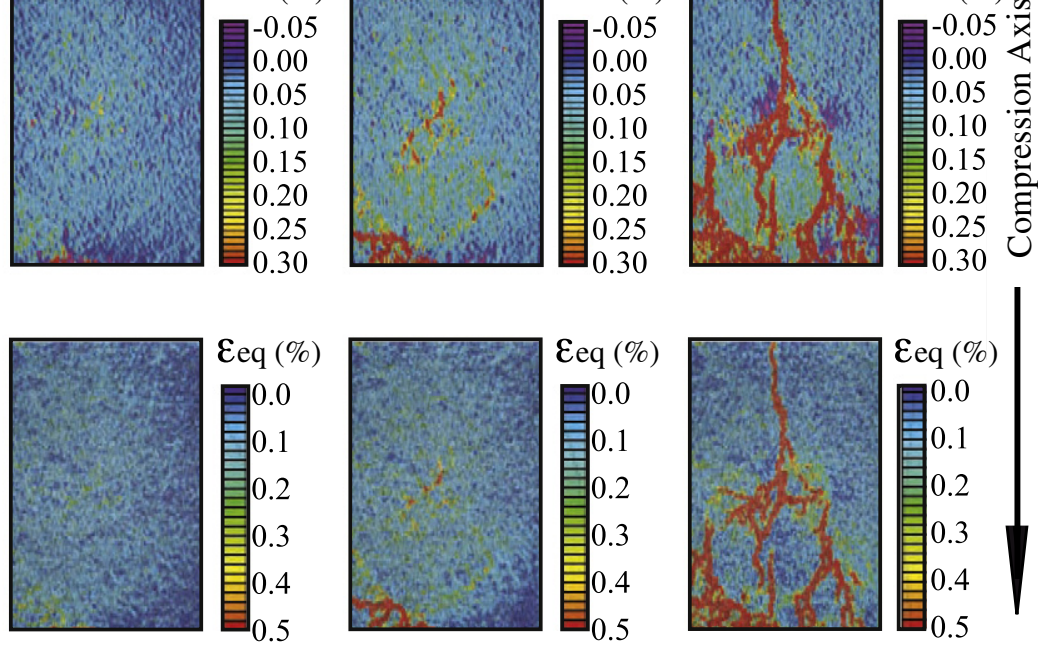

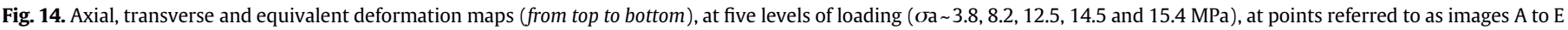

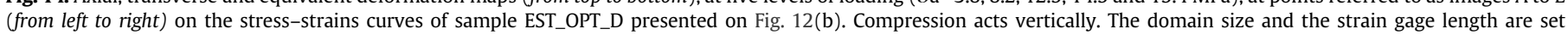
respectively to 50 and 130 pixels. 


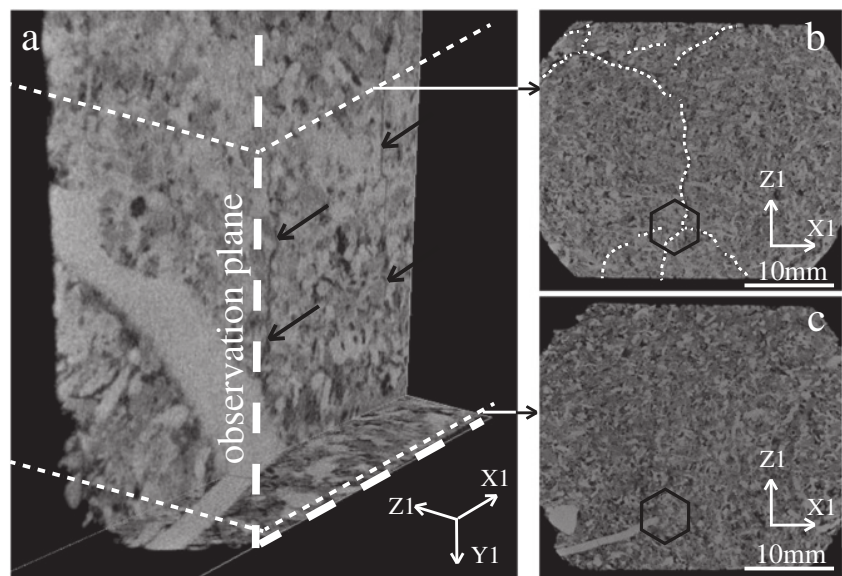

Fig. 15. (a) XR-MCT post-mortem 3D observation of the bottom left part of the sample EST_OPT_D affected by strong strain localization according to the presence of a dense bioclast (appearing in white on the image), localized just behind the in-situ optical observation surface. The observed volume is oriented according to optical axes defined in Fig. 3. (b) 2D slice in the X1Z1 plane, located $6 \mathrm{~mm}$ from the bottom of the sample; dashed lines highlight the location of microcracks. (c) 2D slice in the X1Z1 plane, located $0.25 \mathrm{~mm}$ from the bottom of the sample; the extremity of the bioclast corresponds to the nucleation points of the observed microcracks on (b).

have shown that the sample size has an impact on the Uniaxial Compressive Strength (the smaller the sample size, the higher its strength); probabilistic models of damage also predict such size dependence (Pisarenko and Gland, 2001). At the beginning of the loading a slight out-of-plane tilt of the sample has been recorded, resulting in an apparent low-amplitude extension in the vertical direction (axial component of the deformation). Consequently, some care needs also to be taken for the interpretation of the maps presented in Fig. 14. Contrary to previous observations on the sample EST_OPT_C, the deformation maps (relative to a gage length of 130 pixels $\times 7.5 \mu \mathrm{m}=975 \mu \mathrm{m}$ ) show the emergence of a lens-shape of localized deformation at the bottom of the sample, corresponding to the highly porous zone. A dense bioclast is also identified in this region (see Fig. 4). Before sample failure, while there is no evidence of extension of the deformation lens, the local strains are two times larger at the bottom than in the upper part, where the strains are more diffusively accommodated. Consequently, the failure does not result from a simple shear band propagation, but is characterized by an intense and pervasive crushing of the lower part of the sample extended into a splitting failure in the upper part. The impact of the mesoheterogeneity related to the bioclast is revealed by post-mortem XR-MCT imaging, as shown in Fig. 14. Indeed, the three cracks, observed on the 2D deformation maps on Fig. 14, nucleate in the vicinity of the bioclast (symbolized by the hexagon in Fig. 15b, c) and coalesce at this point into the main vertical fracture. Since this area accommodates large strains before peak stress, it seems pertinent to correlate the initiation of the fracture with this specific heterogeneity. To summarize these observations, one may say that on the one hand, the porosity gradient induces a global gradient of strain accommodation along the observation surface and on the other hand, the presence of the bioclast leads to a more intense strain localization in the left bottom part of the sample and hastens the macroscopic fracturation.

Those comparative experiments highlight more precisely the influence of the three-dimensional distribution of the heterogeneity into the sample:

- on the macroscopic mechanical response, i.e. elastic moduli, mechanical strengths and ultimate strain values, which are highly influenced by the heterogeneity of porosity through a considered sample.

- on the localization of deformation at the mesoscale, which is first controlled by the porosity profile, but may also be strongly

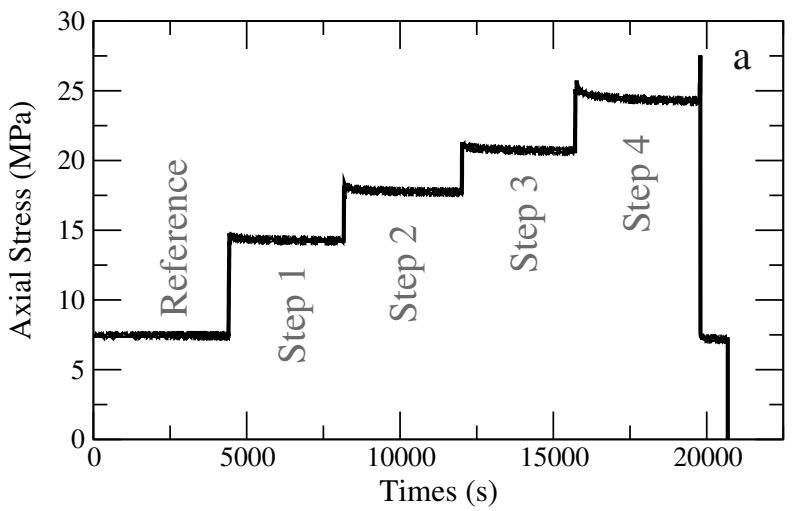

EST_SEM_A

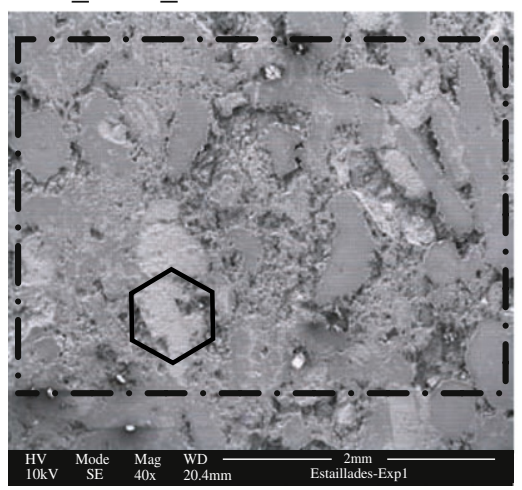

EST_SEM_B

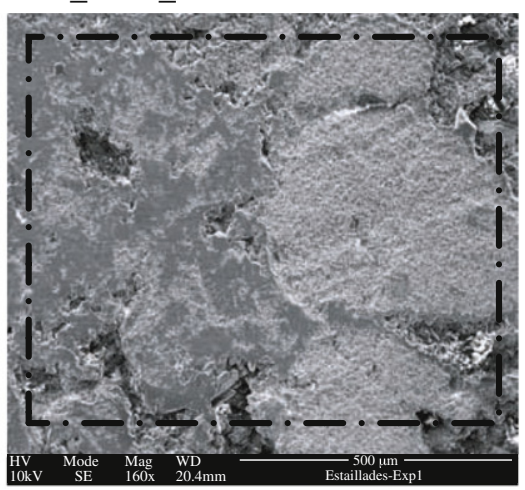

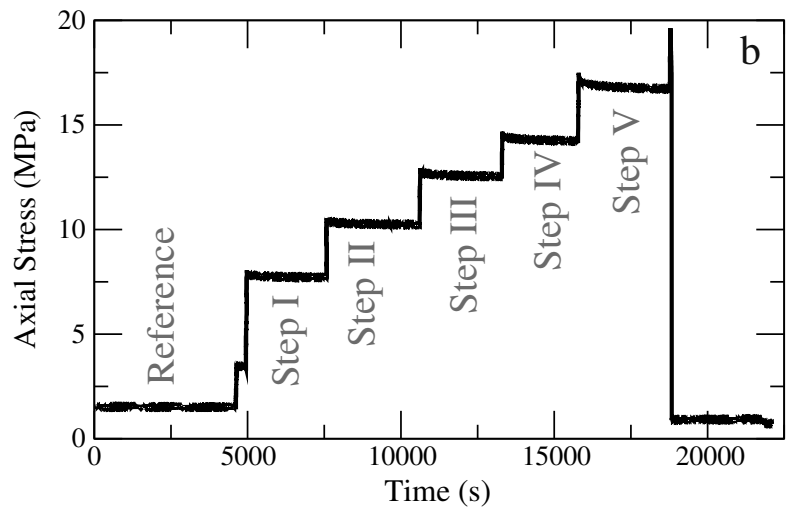

EST_SEM_C

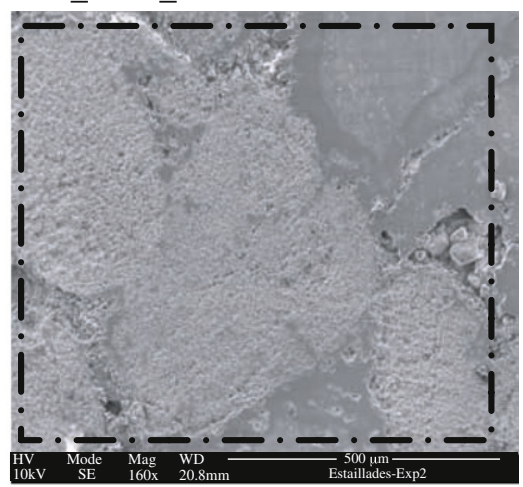

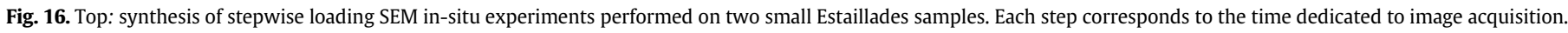

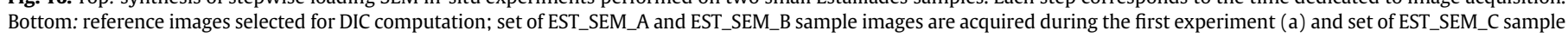
images is acquired during the second experiment (b). Dashed rectangles correspond to the area covered by the grid. 
influenced by the presence of specific heterogeneities, such as bioclasts.

- on the failure mechanisms of this carbonate, which may also differ according to the mesoheterogeneity degree.

Furthermore, we have shown that 2D observations could be strongly influenced by the 3D distribution of local heterogeneity, hence the great interest of performing volumetric investigation by 3D-DIC.

\subsection{Microscopic DIC applied on SEM images}

The objective of the SEM approach is to detect the localized deformation mechanisms, their chronology and the activated mechanisms at the finest scale of the microstructure. The results presented in this section have been obtained on two samples, subjected to incremental axial loading, as reported in Fig. 16. Among the 18 DIC analyses performed on the SEM images, three sequences of images have been selected according to different pertinent scales of measurements and in order to highlight the most important local mechanisms identified on the whole set of deformation maps. The selected reference images, referred to as EST_SEM_A, EST_SEM_B (obtained respectively at magnification $\times 40$ and $\times 160$ during the first experiment) and EST_SEM_C (obtained at magnification $\times 160$ during the second experiment) are also shown in Fig. 16 . EST_SEM_A covers an area of $\sim 4 \times 3 \mathrm{~mm} 2$, comparable to the optical mesoscopic scale, but with a much better image resolution; EST_SEM_B and EST_SEM_C cover an area of $\sim 1 \times 1 \mathrm{~mm} 2$, encompassing only a few aggregates. The results are presented as maps of axial and transverse components of strain and equivalent strain; computed with a gage length of 60 pixels (i.e. $55 \mu \mathrm{m}$ for EST_SEM_A and $14 \mu \mathrm{m}$ for EST_SEM_B and EST_SEM_C), for the images acquired at the axial stresses reported on Fig. 16a and b. On the whole set of images, the compression direction is horizontal.

On the EST_SEM_A set of images, the deformation maps (Fig. 17, steps 2, 3 and 4) clearly show the progressive evolution of the localization of the deformation, which appears at an early stage of compression. This localized deformation is located at the interfaces between dense aggregates, corresponding to weak zones of larger porosity. The local mechanism is essentially the opening of a microcrack in the center of the image, whose tortuous propagation is conditioned by the arrangement of the aggregates. Note also the presence of moiré-like bands in the deformation maps due to systematic errors, which can easily be distinguished from actual localized deformation mechanisms by the fact that these bands move from one image to the other. A large local extensive transverse strain occurring at the interface oriented along the compression direction is the signature of microcrack opening. The evolution of the axial strain shows that orthogonal interfaces are mostly subjected to compressive mechanisms. A few other localized deformation events of lower amplitude appear at the interfaces of dense aggregates on the bottom left part of the image (Fig. 17, step 4) close to the peak stress. No localized deformation event is observed inside the microporous aggregates. DIC applied at this fine scale confirms the previous observations at mesoscale: the propagation of microcracks is not instantaneous, but rather gradual along the regions of weaker strength. We also observe that local relative displacements along cracks are much larger along the tortuous pathway of the interface.
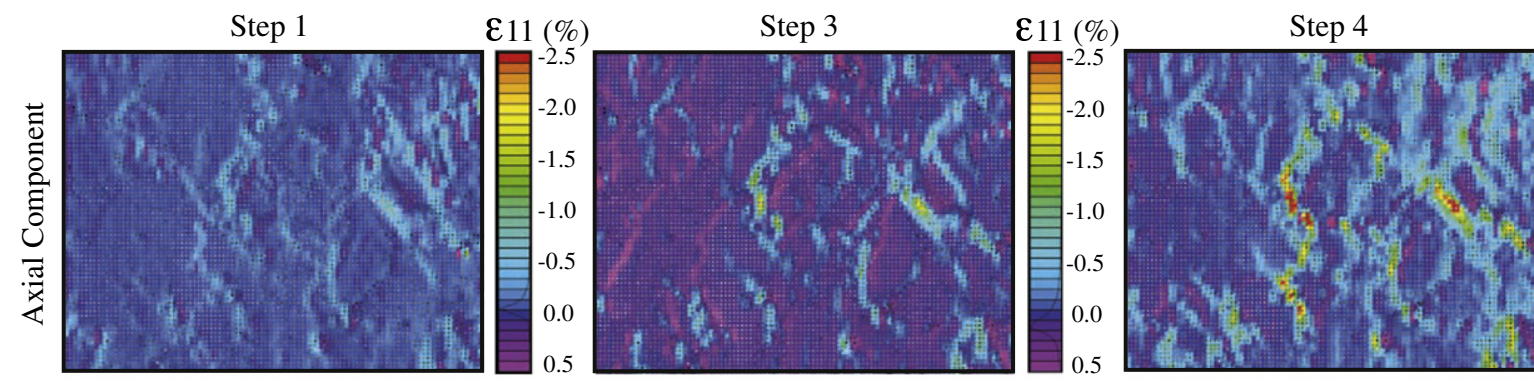

$\varepsilon 11(\%)$

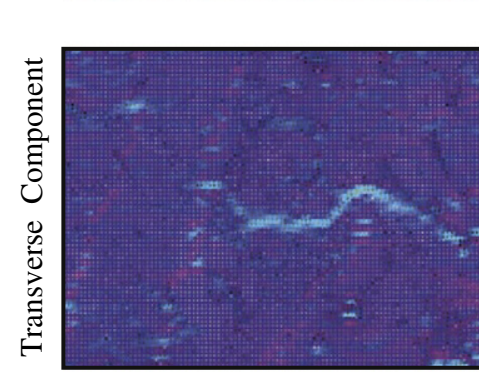

$\varepsilon 22(\%)$
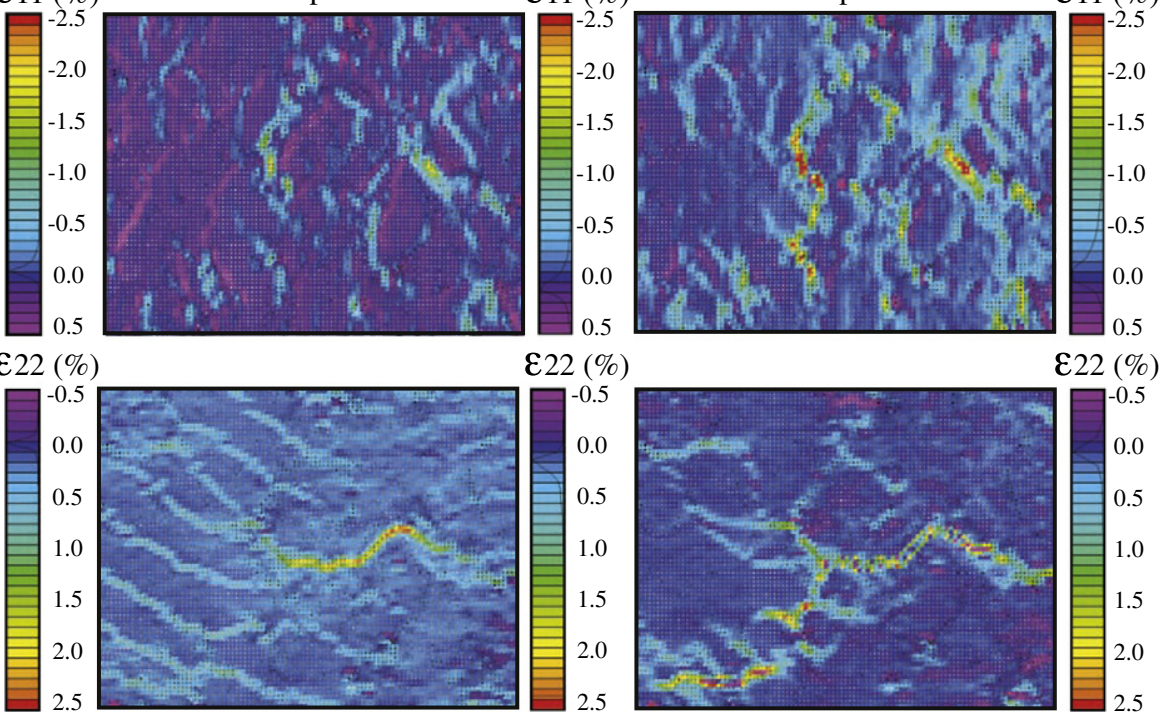

E22 (\%)

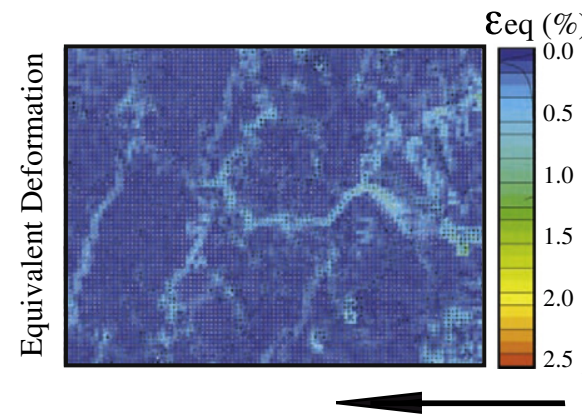

Eeq (\%)
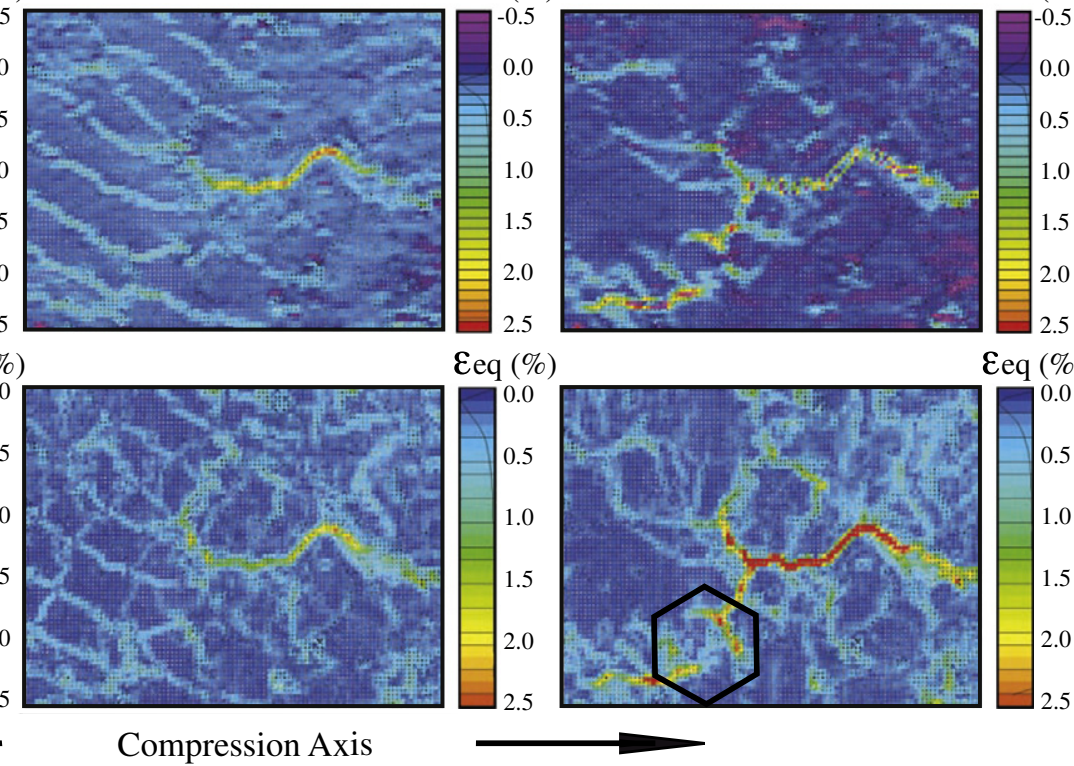

Eeq (\%)

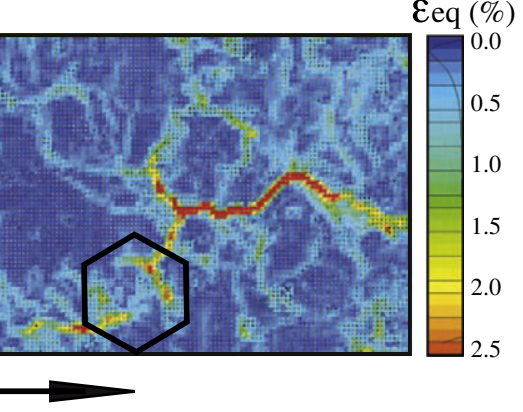

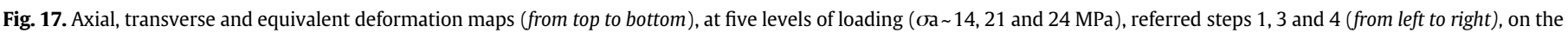

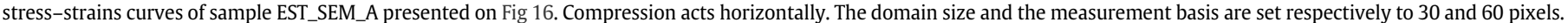


Moreover, the strong stress relaxation observed during the last loading step (Fig. 17, step 4), is associated with a large opening of a crack in the central area, where the local strains exceed $10 \%$, corresponding to a relative displacement of the crack lips of approximately 6 pixels, which initiates the branching of the final failure. At this scale, the principal crack seems to not propagate through the encountered microporous aggregate (hexagonal region in Fig. 17, image 4), while another crack is opening on the other side.

The considered region EST_SEM_B is constituted of microporous aggregates on the right side and dense aggregates on the left side. The largest localized deformation in this region is characterized by the opening of a crack in the more porous zone at the interfaces between the different kinds of aggregates (right bottom part on Fig. 18, step 1). We also observe localized deformation events of smaller amplitude, taking place along the interfaces oriented in the compression direction. A non-negligible compactant mechanism appears also inside the large microporous aggregate, along a pre-existing structure of higher porosity (Fig. 18, step 1). These local pre-existing zones inside the microporous aggregates are common and their closure takes place only when their trace appears perpendicular to the loading direction.

An example of diffusive strain accommodation affecting the microporous aggregates is shown in Fig. 19. The EST_SEM_C image (Fig. 16) is composed of a large central microporous aggregate showing a tenuous crossing crack oblique to the axial stress direction, in contact with a dense aggregate showing a wide crack (on the top right of the image). At early loading, $\sigma<8 \mathrm{MPa}$, a first compactant mechanism appears at the interface in the microporous aggregates (on the bottom left), normal to the direction of compression. At higher compression, $\sigma \sim 12 \mathrm{MPa}$, simultaneously, the weak zone in the dense aggregates is activated (on the top right) and the interface between microporous aggregates starts to slide (on the top). During all the loading, the central microporous aggregate, despite its thin crossing crack, does not show any evidence of localization of the deformation.

The analysis of the deformation maps superimposed over the microstructure highlights the strong impact of the microheterogeneity on the localization of the deformation. As revealed by mesoscopic DIC, sample failure results of gradual localized deformation events take place in the weaker zones of the microstructure. Indeed, the most porous inter-aggregates interfaces show early activations, depending on their orientation with respect to the loading direction. We distinguish compressive and dilatant mechanisms affecting interfaces respectively oriented orthogonally and along the axial loading direction, which are characteristic of the opening and the diffusive propagation of cracks. Furthermore, only pre-existing weak structures in the dense aggregates exhibit both compressive and dilatant mechanisms. Conversely, no localized deformation is recorded inside the microporous aggregates, with the exception of specific preexisting inhomogeneities or microdefects transverse to the axial loading direction. Consistently with previous post-mortem microstructural observations (Dautriat et al., submitted for publication), it appears that the microporous aggregates structure is very likely the factor, which leads to a diffusive accommodation of the deformation. Consequently, those microstructural components contribute to maintain the integrity of the sample during compression.
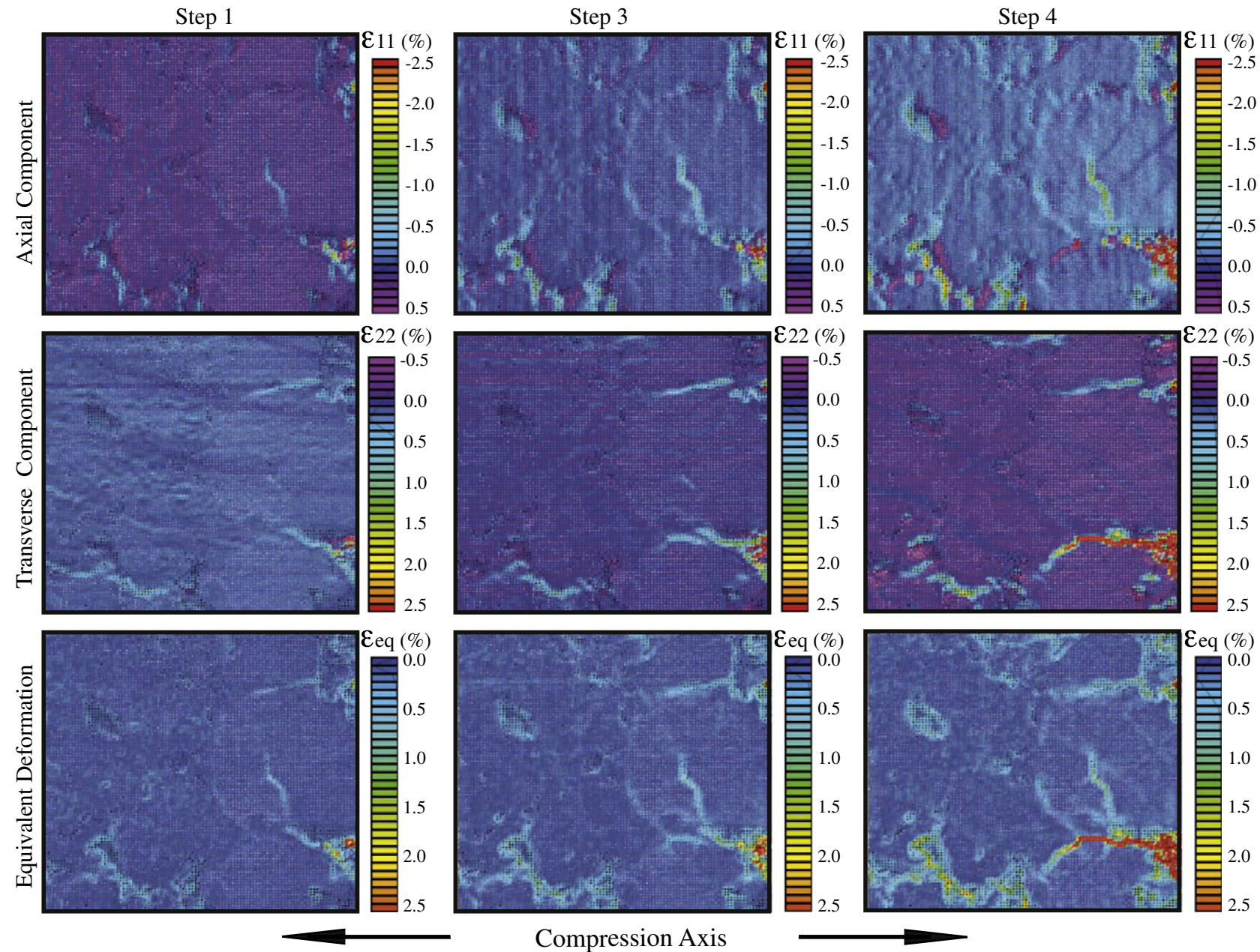

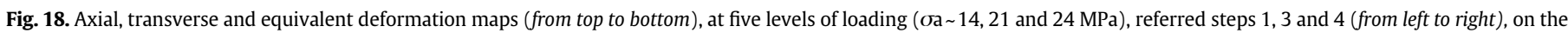

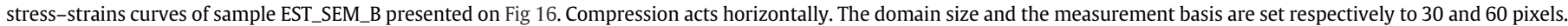



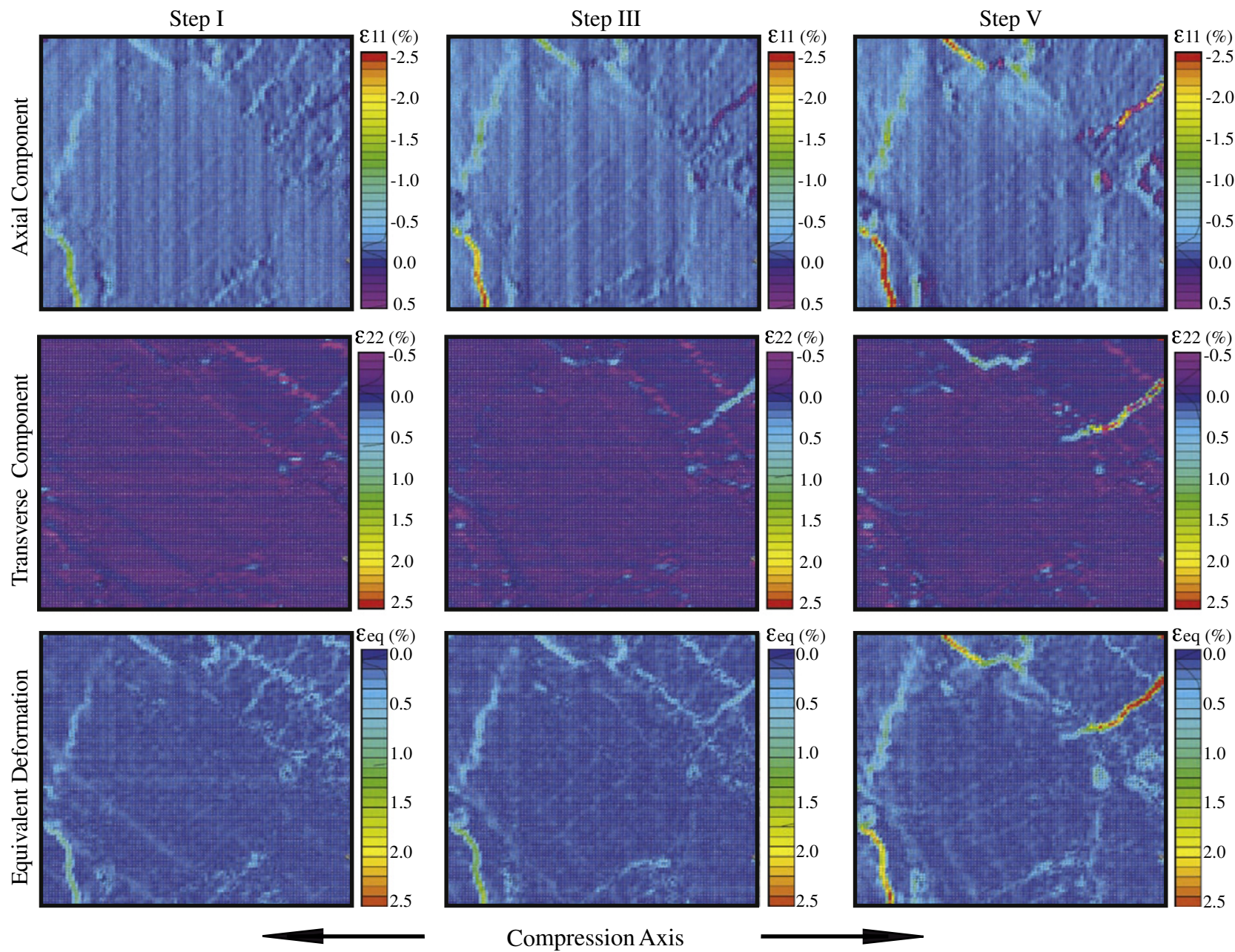

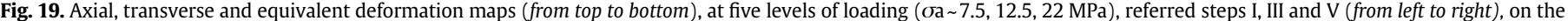

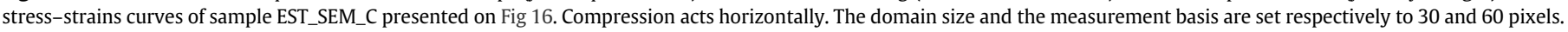

\section{Conclusions}

We have assessed and successfully applied the 2D-DIC method at different scales of observation to the Estaillades limestone, a heterogeneous carbonate, submitted to simple compression tests. The challenging very small deformation sustained by this material at failure made it crucial to optimize the quality of the images by improving the local contrast, and also by suitably tuning the correlation parameters (grid, correlation domain sizes and gage length relative to local strains), so that an optimum could be achieved between accuracy on displacement field computation and quantitative precision on activation mechanisms. The uncertainties of the DIC method have been considerably reduced thanks to the quality of the new generation of imaging devices used in this study. The experimental methodology, combining both optical and SEM imaging, allows to detect the various deformation and damage mechanisms and to understand their sequential activity, from the macroscopic sample scale down to the microstructure scale. At different scales of optical observation, the DIC computations provide consistent results and give a correct estimation of global deformations. We show on the basis of the image processing that the mesoscopic scale heterogeneity, which is essentially resulting from local fluctuations of porosity gradients and distribution of more or less dense aggregates, is directly responsible for the early onset of localization, as well as for the development of a network of microcracks. At the scales investigated by SEM, the local micromechanisms of deformation are clearly revealed by the full-field DIC measurements, whilst they are not identified by direct image observations. In agreement with the preliminary conclusions reported in a previous work (Dautriat et al., submitted for publication) and based on post-mortem analysis, our in-situ observations demonstrate a diffuse strain accommodation in the microporous aggregates and a gradual opening of microcracks in the most porous zones, i.e. at the inter-aggregates interface and at some pre-existing discontinuities within the dense aggregates. Independently of the scale of observation, the localized damage and the final macroscopic failure of the samples do not appear as spontaneous events. Damage happens gradually, with an early occurrence of microcracks, followed by slow opening and growth, until a critical level of microcrack coalescence, leading to failure.

Obviously, the representativeness of results obtained by 2D surface observations remains debatable. In order to clarify this point, 3D DIC coupled with microtomography and in-situ compression would be required (Lenoir et al., 2007). But for now, this can only be done at the cost of a downgraded spatial resolution. For instance, Bésuelle et al. (2006) obtained voxels sizes of $\sim 14 \mu \mathrm{m} 3$ on macroscopic clay-rock samples, which is not sufficient to observe the micromechanisms taking place in carbonates. On much smaller samples, one may reach voxels sizes of less than $1 \mu \mathrm{m} 3$ under axial or hydrostatic loading conditions (Adrien et al., 2007). But, in order to be representative, the sample size has to be selected depending on the structural heterogeneities of the considered material, which in our case precludes very small specimens. 


\section{Acknowledgments}

At LMS, the authors are grateful to Daniel Caldemaison, Pierre Valli and Diansen Yang for technical assistance with the SEM apparatus, testing machine and optical set-ups respectively. At IFP, we warmly thank Corinne Fichen, Jean Guelard and Valérie Poitrineau, respectively for the X-Ray microtomography imaging, the petrophysical characterization and the UCS tests. The SEM investigations have been performed on the environmental FEG-ESEM FEI Quanta 600 of LMS which has been funded with the help of the SESAME program of Îlede-France region, École Polytechnique ParisTech and CNRS. The optical devices used at LMS for the macroscopic and mesoscopic investigations were funded with the help of CNRS and ANDRA in the context of micromechanical investigations on clay-rocks.

\section{References}

Adam, J., Urai, J.L., Wieneke, B., Oncken, O., Pfeiffer, K., Kukowski, N., Lohrmann, J., Hoth, S., Van der Zee, W., Schmatz, J., 2005. Shear localisation and strain distribution during tectonic faulting - new insights from granular-flow experiments and highresolution optical image correlation techniques. J. Struct. Geol. 27 (2), 283-301.

Adrien, J., Maire, E., Gimenez, N., Sauvant-Moynot, V., 2007. Experimental study of the compression behavior of syntactic foams by in situ X-ray tomography. Acta Mater. 55 (5), 1667-1679.

Allais, L., Bornert, M., Bretheau, T., Caldemaison, D., 1994. Experimental characterization of the local strain field in a heterogeneous elastoplastic material. Acta Metall. Mater. 42 (11), 3865-3880.

Bandhari, A.R., Indue, J., 2005. Experimental study of strain rates effects on localization characteristics of soft rocks. Soil Found. 45 (1), 125-140.

Bay, B.K., Smith, T.S., Fyrhie, D.P., Saad, M., 1999. Digital volume correlation: threedimensional strain mapping using X-ray tomography. Exp. Mech. 39 (3), 217-226.

Bésuelle, P., Viggiani, G., Lenoir, N., Desrues, J., Bornert, M., 2006. X-ray micro CT for studying strain localization in clay rocks under triaxial compression. Advances in Xray Tomography for Geomaterials. ISTE Ltd, pp. 35-52.

Bornert, M., Chaix, J.M., Doumalin, P., Dupré, J.C., Fournel, T., Jeulin, D., Maire, E., Moreaud, M., Moulinec, H., 2004. Mesure tridimensionnelle de champs cinématiques par imagerie volumique pour l'analyse des matériaux et des structures. Instrum. Mesure Métrologie 4, 43-88 (In french).

Bornert, M., Brémand, F., Doumalin, P., Dupré, J.-C., Fazzini, M., Grédiac, M., Hild, F., Mistou, S., Molimard, J., Orteu, J.-J., Robert, L., Surrel, Y., Vacher, P., Wattrisse, B., 2009. Assessment of digital image correlation measurement errors: methodology and results. Exp. Mech. 49 (3), 353-370.

Bornert, M., Valès, F., Gharbi, H., Nguyen Minh, D., 2010. Multiscale full-field strain measurements for micromechanical behaviour of clayey rocks. Strain 46 (1), 33-46 (14).

Chu, T., Ranson, W., Sutton, M., Peters, W., 1985. Applications of DIC techniques to experimental mechanics. Exp. Mech. 25, 232-244.

CorrelManuV., 2005. Doc. available from: <http://www.lms.polytechnique.fr/users/ bornert/cmv_14/doc/>.

Dautriat, J., Gland, N., Guelard, J., Dimanov, A., Raphanel, J.L., 2009. Axial and radial permeability evolutions of compressed sandstones: end effects and shear-band induced permeability anisotropy. Pure Appl. Geophys. 166 (5-7), 1037-1061.

Dautriat, J., Gland, N., Dimanov, A., Raphanel, J.L., in press. Hydromechanical behavior of carbonate rock: the effects of stress path and heterogeneity. J. Geophys. Res.

Doumalin, P., 2000. Microextensiométrie par corrélation d'images. Thèse de Doctorat, Ecole Polytechnique, France. (In french).

Doumalin, P., Bornert, M., 2000. Micromechanical applications of digital image correlation techniques. In: Jacquot, P., Fournier, J.M. (Eds.), Proc. of Interferometry in Speckle Light: Theory and Applications. Springer, pp. 67-74.

Doumalin, P., Bornert, M., Caldemaison, D., 1999. Microextensometry by image correlation applied to micromechanical studies using the Scanning Electron Microscopy. Exp. Mech Proc. Int. Conf. Advanced Technol, pp. 81-86. Ube City, Japan.

Doumalin, P., Bornert, M., Crépin, J., 2003. Caractérisation de la répartition de la déformation dans les matériaux hétérogènes. Mécanique Ind. 4, 607-617 (In french).

Elbachiri, K., Doumalin, P., Crépin, J., Bornert, M., Barberis, P., Rebeyrolle, V., Bretheau, T., 2008. Characterization of local strain distribution in Zircaloy-4 and M5 $₫$ alloys. J. ASTM 5 (9), 1-11.

Germaneau, A., Doumalin, P., Dupre, J.C., 2007. Full 3D measurement of strain field by scattered light for analysis of structures. Exp. Mech. 47 (4), 523-532.

Grédiac, M., 2004. The use of full-field measurement methods in composite material characterization: interest and limitations. Compos. Part. A 35, 751-761.
Gudehus, G., Nübel, K., 2004. Evolution of shear bands in sand. Geotechnique 54 (3), 187-201.

Hall, S.A., Muir Wood, D., Ibraim, E., Viggiani, G., 2009. Localized deformation patterning in 2D granular materials revealed by digital image correlation. Granular Matter 12 (1), $1-14$.

Héripré, E., Dexet, M., Crépin, J., Gélébart, L., Roos, A., Bornert, M., Caldemaison, D., 2007 Coupling between experimental measurements and polycrystal finite element calculations for micromechanical study of metallic materials. Int. J. Plast. 23 (9), 1512-1539.

Hild, F., Maire, E., Roux, S., Witz, J.-F., 2009. Three-dimensional analysis of a compression test on stone wool. Acta Mater. 57 (11), 3310-3320.

Hoek, E., Brown, E.T., 1980. Underground Excavations in Rock. The Institution of Mining and Metallurgy, London.

Huon, V., Wattrisse, B., El Youssoufi, M.S., Chrysochoos, A., 2007. Elastic behavior of anisotropic terra cotta ceramics determined by kinematic full-field measurements. J. Ceram. Soc. 27, 2303-2310.

Janesick, J.R., 2001. Scientific Charge-Coupled Devices. Society of Photo-Optical Instrumentation Engineers. Press Monogram PM83, Washington.

Lenoir, N., Bornert, M., Desrues, J., Bésuelle, P., Viggiani, G., 2007. Volumetric digital image correlation applied to X-ray microtomography images from triaxial compression tests on argillaceous rocks. Strain 43 (3), 193-205.

Louis, L., Wong, T.-F., Baud, P., 2007. Imaging strain localization by X-ray radiography and digital image correlation: deformation bands in Rothbach sandstone. J. Struct. Geol. 29 (1), 129-140.

Marya, M., Hector, L.G., Verma, R., Tong, W., 2006. Microstructural effects of AZ31 magnesium alloy on its tensile deformation and failure behaviors. Mat. Sci. Eng. A 418 (1-2), 341-356

NGuyen, T.L., Hall, S.A., Vacher, P., Viggiani, G., 2011. Fracture mechanisms in soft rock: Identification and quantification of evolving displacement discontinuities by extended digital image correlation. Tectonophysics. 503 (1-2), 117-128 (this issue).

Paterson, M.S., Wong, T.-F., 2005. Experimental Rock Deformation - The Brittle Field 2nd Edition. Springer, New York.

Peters, W., Ranson, W., 1982. Digital imaging techniques in experimental stress analysis. Opt. Eng. 21 (3), 427-431.

Pires, G.P., Robert, M.H., Arrieux, R., 2004. Studies on drawing of the aluminium A5052 alloy in thixocast condition. J. Mater. Process. Technol. 157-158, 596-603.

Pisarenko, D., Gland, N., 2001. Modeling of scale effects of damage in cemented granular rocks. Phys. Chem. Earth A. 26 (1-2), 83-88.

Racine, A., 2005. Influence de l'orientation des hydrures sur les modes de déformation, d'endommagement et de rupture du Zircaloy-4 hydruré, PhD thesis, Ecole Polytechnique, Palaiseau, France. (In French).

Rae, P.J., Palmer, S.J.P., Golrein, H.T., Lewis, A.L., Field, J.E., 2004. White-light digital image cross-correlation (DICC) analysis of the deformation of composite materials with random microstructure. Opt. Lasers Eng. 41 (4), 635-648.

Rechenmacher, A.L., 2006. Grain-scale processes governing shear band initiation and evolution in sands. J. Mech. Phys. Solids 54 (1), 22-45.

Rechenmacher, A.L., Finno, R.J., 2004. Digital image correlation to evaluate shear banding in dilative sands. Geotech. Test. J. 27 (1), 13-22.

Roux, S., Hild, F., 2006. Stress intensity factor measurements from digital image correlation: post-processing and integrated approaches. Int. J. Fract. 140, 141-157.

Schreier, H., Sutton, M., 2002. Systematic errors in digital image correlation due to undermatched subset shape functions. Exp. Mech. 43 (3), 303-311.

Schreier, H., Braasch, J., Sutton, M., 2000. Systematic errors in digital image correlation caused by intensity interpolation. Opt. Eng. 39 (11), 2915-2921.

Schreier, H., Garcia, D., Sutton, M., 2004. Advances in light microscope stereo vision. Exp. Mech. 44, 278-288.

Sutton, M., Wolters, W., Peters, W., McNiell, S., 1983. Determination of displacements using an improved digital correlation method. Image Visual. Comput. 1, 133-139.

Sutton, M., Yan, J., Tiwari, V., Schreier, H., Orteu, J.-J., 2008. The effect of out-of-plane motion on 2D and 3D digital image correlation measurements. Opt. Lasers Eng. 46, 746-757.

Sutton, M., Orteu, J.-J., Schreier, H., 2009. Image Correlation for Shape, Motion and Deformation Measurements: Basic Concepts, Theory and Applications. Springer New York.

Tatschl, A., Kolednik, O., 2003. On the experimental characterization of crystal plasticity in polycrystals. Mater. Sci. Eng. A 342, 152-168.

Vacher, P., Dumoulin, S., Morestin, F., Mguil-Touchal, S., 1999. Bidimensional strain measurement using digital images. J. Mech. Eng. Sci. C 213, 811-817.

Vasarhelyi, B., 2003. Some observations regarding the strength and deformability of sandstones in dry and saturated condition. Bull. Eng. Geol. Environ. 62, 245-249.

Viggiani, G., Hall, S.A., 2008. Full-field measurements, a new tool for laboratory experimental geomechanics. In: Burns, S.E., Mayne, P.W., Santamarina, J.C. (Eds.) Deformation Characteristics of Geomaterials, 1. IOS Press, pp. 3-26.

Wattrisse, B., Chrysochoos, A., Muracciole, J.-M., Némoz-Gaillard, M., 2001. Analysis of strain localization during tensile tests by digital image correlation. Exp. Mech. 41 (1), 29-39.

Yang, D.S., Bornert, M., Gharbi, H., Valli, P., Wang, L.L., 2010. Optimized optical setup for DIC in rock mechanics. Proc. 14th Int. Conf. Exp. Mech., Poitiers, France. 